\title{
Crosstalk of multi-omics reveals specific characteristics in active ulcerative colitis patients with depression and anxiety
}

\section{Xiaomin Yuan}

Affiliated Hospital of Nanjing University of Chinese Medicine https://orcid.org/0000-0001-8592-6520

\section{Biqing Chen}

Affiliated hospital of Nanjing University of Chinese Medicine

\section{Zhenglan Duan}

Affiliated Hospital of Nanjing University of Chinese Medicine

\section{Ziqian Xia}

Affiliated hospital of Nanjing University of Chinese Medicine

\section{Yang Ding}

Affiliated Hospital of Nanjing University of Chinese Medicine

\section{Tuo Chen}

Affiliated Hospital of Nanjing University of Chinese Medicine

\section{Huize Liu}

Affiliated Hospital of Nanjing University of Chinese Medicine

\section{Baosheng Wang}

Affiliated Hospital of Nanjing Univerity of Chinese Medicine

\section{Bolin Yang}

Affiliated Hospital of Nanjing University of Chinese Medicine

\section{Xiaoyong Wang}

Affiliated Hospital of Nanjing University of Chinese Medicine

\section{Shijia Liu}

Affiliated Hospital of Nanjing University of Chinese Medicine

Jin-Yong Zhou

Affiliated Hospital of Nanjing University of Chinese Medicine

Yajun Liu

Affiliated Hospital of Nanjing University of Chinese Medicine

\section{Zhaofeng Shen}

Affiliated Hospital of Nanjing University of Chinese Medicine

\section{Jun Xiao}

Affiliated Hospital of Nanjing University of Chinese Medicine

Hongtao Shang

Affiliated Hospital of Nanjing University of Chinese Medicine

\section{Weiwei Liu}

Affiliated Hospital of Nanjing University of Chinese Medicine

Guoping Shi ( $\nabla$ sgp-2020@outlook.com ) 
Affiliated Hospital of Nanjing University of Chinese Medicine

\section{Lei Zhu ( $\nabla$ zhulei5100@163.com )}

Affiliated Hospital of Nanjing University of Chinese Medicine

Yugen Chen ( $\square$ yugen.chen@njucm.edu.cn )

Affiliated Hospital of Nanjing University of Chinese Medicine

\section{Research}

Keywords: Ulcerative colitis, Microbiota-gut-brain axis, Metabolomics, Proteomics, Multi-omics analysis

Posted Date: August 2nd, 2020

DOI: https://doi.org/10.21203/rs.3.rs-49500/v1

License: (c) This work is licensed under a Creative Commons Attribution 4.0 International License. Read Full License

Version of Record: A version of this preprint was published at American Journal of Gastroenterology on October 1st, 2020. See the published version at https://doi.org/10.14309/01.ajg.0000702852.34775.fa. 


\section{Abstract}

Background:

Ulcerative colitis (UC) patients have a high incidence of mental disorders. The microbiota-gut-brain axis dysfunction is considered as one important pathogenesis of mental diseases. At present, little is known about how gut microbiota interact with the host in UC patients with depression and anxiety.

Results: This prospective observational study enrolled 240 Chinese patients in two cohorts: the discovery cohort, including 69 active UC patients, 49 non-inflammatory bowel disease (IBD) depression and anxiety patients, and 62 healthy people, and the replication cohort of 60 active UC patients. About half of active UC patients showed symptoms of depression or anxiety. Through 16s rRNA sequencing, it was found that these UC patients accompanied with depression and anxiety had lower fecal microbial abundance with more Sellimonas , Eubacterium ventriosum group , Enterococcus , and Peptoclostridium , but less Prevotella_9 , Erysipelotrichaceae UCG_003 , Collinsella , and Dorea , compared with non-depressed/ anxious UC patients. Serum metabolome and proteome analysed using liquid chromatography/ mass spectrometry showed significantly increased glycochenodeoxycholate, stearoyllysophosphatidylcholine, and glyceryl stearates, while decreased 2'-deoxy-D-ribose and a set of immunoglobulin protein in the serum of UC patients with depression and anxiety. Through integration of multiple statistical analyses and multi-omics correlation analyses, we revealed a highly connected and comprehensive network, centring on Prevotella_9 and 1-stearoyl-sn-glycerol, composed of these bacteria, metabolites and proteins associated with UC-specific depression and anxiety.

Conclusion: This study has identified a highly connected multi-omics network, composed of a set of gut microbiota, serum metabolites and proteins, specifically related to depression and anxiety in active UC. This network might influence host's mental state through mediating the effect of gut inflammation on synapse pruning in the central nervous system.

\section{Background}

Ulcerative colitis (UC) is one kind of chronic and recurrent inflammatory bowel disease (IBD) occurred in colon with long-term relapse and remission. UC has a rapidly increasing incidence rate in the developing countries[1]. While the aetiology of UC remains not thoroughly understood, one potential cause could be an aggravated immune response towards the gut microbiota in genetically susceptible individuals[2].

The incidence of mental disorders, such as depression and anxiety, is significantly higher in UC patients than in healthy people[3,4,5].The comprehensive prevalence of depression and anxiety in UC patients is about $16.7 \%$ and $28.3 \%$, respectively, which is higher when UC patients are in an active stage, that is, $40.7 \%$ depression and $75.6 \%$ anxiety[6]. There is mutual influence for UC and mental disorders[7]. On one hand, the occurrence of mental disorders is affected by UC-related factors, such as disease activity, complicated conditions (active inflammation, nutrition, and surgical problems), complex extra-intestinal manifestations, and reduced quality of life[3,8,9]. Depression could be improved by treatment designated for UC[10]. On the other hand, mental disorders could lead to poor prognosis and increased risk of UC. Depression and anxiety at baseline are related to more frequent disease flare, resulting in treatment escalation, poor compliance, increased hospitalization and surgical risks[7,11,12]. Longterm depression and anxiety will reduce the quality of life and cost many medical resources. Antidepressant drugs could effectively reduce the disease activity and gastrointestinal symptoms of UC[13]. However, clinicians have not paid enough attention to evaluation and treatment of mental disorders in UC patients although suggested by the 
latest American College of Gastroenterology nursing guidelines[14]. About one third of depression and two thirds of anxiety in UC patients have not been diagnosed[15], and most UC patients have not received adequate and efficient psychological counseling or treatment in time. Therefore, a comprehensive understanding of the fundamental mechanism of UC-related mental disorders is of great clinical significance for discovering new potential therapeutic targets to treat UC-related comorbidities and improve quality of life for UC patients.

In recent years, the microbiota-gut-brain axis has been revealed to play a complex and important role in the development of mental diseases such as depression and anxiety. Gut microbiota and central nervous system interplayed with each other, through the immune system and metabolites in the blood[16,17]. Gut microbiota can produce certain neurotransmitters such as dopamine[18], serotonin[19], and $y$-aminobutyric acid (GABA)[20], well known for affecting mood and cognition. Gut microbiota and the related metabolome imbalance could be one crucial bridge in the pathogenesis of depression and anxiety.

An animal study showed that biogenic lactic acid bacterium prevented IBD-like pathology and depression-like behavior in mice via decreasing the levels of rectal and hippocampal inflammatory cytokines[21]. Currently, there is only one clinical study that has investigated the relationship between gut microbiota and mental states in IBD patients at remission stage by $16 \mathrm{~S}$ rRNA sequencing of the feces[22], while little is known about the situations in IBD patients at active stage (which is more severe and needs more attention) and its underlying mechanisms.

In this study, the characteristics of UC-related depression and anxiety was investigated through a multi-omics approach, integrating gut microbiota, serum metabolome, and proteome (Fig.1). 69 active UC patients were recruited in the discovery cohort, with their depression and anxiety levels evaluated by standard questionnaires and their fecal 16S rRNA V3-V4 regions sequenced. Specific composition of gut microbiota was found to be associated with the depression and anxiety states in the discovery cohort and further replicated in another cohort of 60 active UC patients (the replication cohort). These gut microbiota features were specific to depression and anxiety associated with UC, as they were unrelated to depression without IBD or UC itself. Serum metabolome and proteome of the replication cohort were further profiled to explore the potential pathways between gut microbiota and brain. This study suggests an association between depression and anxiety states in UC patients with a microbiota-metaboliteprotein interaction network, including Prevotella, 1-Stearoyl-sn-glycerol, and a set of immune-related proteins. The findings would help polishing the auxiliary diagnosis and accurate treatment of depression and anxiety association with UC.

\section{Results}

\section{General characteristics in the study cohorts}

A total of 240 participants were enrolled, divided into four groups, UC discovery group ( $n=69)$, UC replication group $(n=60)$, non-IBD depression and anxiety group (MDD, $n=49)$, and healthy control group $(H C, n=62)$. Except body mass index, there is no significant difference in the general information among the studied groups (Table 1, Additional file 2: Table S1).

\section{Table 1 Summary statistics of the studied cohorts}




\begin{tabular}{|c|c|c|c|c|c|}
\hline Characteristics & $\begin{array}{l}\text { UC Discovery } \\
(n=69)\end{array}$ & $\begin{array}{l}\text { UC Replication } \\
(n=60)\end{array}$ & $\begin{array}{l}\text { MDD } \\
(n=49)\end{array}$ & $\begin{array}{l}\mathrm{HC} \\
(n=62)\end{array}$ & $p$-value \\
\hline Female $^{\#}$ & $37(53.6)$ & $28(46.7)$ & $29(59.2)$ & $36(58.1)$ & $0.51^{\mathrm{a}}$ \\
\hline $\mathrm{Age}^{\$}$ & $40(31,50)$ & $40.5(30,52.5)$ & $\begin{array}{l}35(25,50.5) \\
n=48\end{array}$ & $42(30,53)$ & $0.32^{\mathrm{b}}$ \\
\hline \multirow[t]{2}{*}{$\mathrm{BM} \mathrm{I}^{\$}$} & 21.3 & 21.2 & 22.3 & 23.4 & $<0.001^{b}$ \\
\hline & $(19.2,23.4)$ & $(19.1,23.5)$ & $\begin{array}{l}(19.9,24.0) \\
n=44\end{array}$ & $(21.5,25.1)$ & \\
\hline \multirow[t]{2}{*}{ Smokers ${ }^{\#}$} & $9(13.0)$ & $10(16.7)$ & $6(13.3)$ & $5(9.6)$ & $0.56^{\mathrm{a}}$ \\
\hline & & & $\mathrm{n}=45$ & & \\
\hline \multirow[t]{2}{*}{ Alcohol Consumers } & $8(11.6)$ & $6(10.0)$ & $6(13.3)$ & 11(17.7) & $0.61^{\mathrm{a}}$ \\
\hline & & & $\mathrm{n}=45$ & & \\
\hline Depression\# & $38(55.1)$ & $37(61.7)$ & $49(100)$ & $0(0)$ & $<0.001^{\mathrm{a}}$ \\
\hline Anxiety\# & $33(47.8)$ & $32(53.3)$ & $49(100)$ & $0(0)$ & $<0.001^{\mathrm{a}}$ \\
\hline PHQ-9* & $6.4 \pm 5.3$ & $7.6 \pm 6.3$ & $12.5 \pm 5.4$ & $1.5 \pm 1.6$ & $<0.001^{b}$ \\
\hline GAD-7 ${ }^{*}$ & $5.1 \pm 4.4$ & $5.9 \pm 5.1$ & $11.2 \pm 4.7$ & $1.3 \pm 1.5$ & $<0.001^{b}$ \\
\hline UC history $\$$ & $3(1,5)$ & $3.5(1,6)$ & - & - & $0.76^{\mathrm{C}}$ \\
\hline Disease extent ${ }^{\#}$ & & & & & $0.40^{c}$ \\
\hline Proctitis & $14(20.3)$ & $14(23.3)$ & - & - & \\
\hline Left-sided colitis & $16(23.9)$ & $17(28.3)$ & - & - & \\
\hline Pancolitis & $39(56.5)$ & $29(48.3)$ & - & - & \\
\hline \multicolumn{6}{|l|}{ Medication ${ }^{\#}$} \\
\hline 5-ASA & $50(72.5)$ & $45(75)$ & - & - & $0.90^{\mathrm{a}}$ \\
\hline Antibiotics & $2(2.9)$ & $3(5.0)$ & - & - & $0.66^{\mathrm{a}}$ \\
\hline Probiotics & $25(36.2)$ & $25(41.7)$ & - & - & $0.65^{\mathrm{a}}$ \\
\hline Enema & 13(18.8) & 10(16.7) & - & - & $0.93^{\mathrm{a}}$ \\
\hline None & $4(5.8)$ & $3(5.0)$ & - & - & $1.00^{\mathrm{a}}$ \\
\hline Mayo score ${ }^{*}$ & $6.5 \pm 2.4$ & $6.5 \pm 2.8$ & - & - & $0.90^{\mathrm{C}}$ \\
\hline \multicolumn{6}{|l|}{ Laboratory data\$ } \\
\hline Faecal calprotectin $(\mu \mathrm{g} / \mathrm{g})$ & $\begin{array}{l}371.8(66.4,830.2) \\
n=53\end{array}$ & $\begin{array}{l}326.8(55.3,891.8) \\
n=51\end{array}$ & - & - & $1.00^{\mathrm{C}}$ \\
\hline
\end{tabular}




\begin{tabular}{|lllll|}
$\begin{array}{l}\text { Erythrocytesedimentationrate } \\
\text { (mm/hour) }\end{array}$ & $\begin{array}{l}6(3,24) \\
\mathrm{n}=47\end{array}$ & $\begin{array}{l}10(5,17) \\
\mathrm{n}=57\end{array}$ & - & $1.00^{\mathrm{c}}$ \\
C-reactive protein (mg/L) & $\begin{array}{l}2.24(1.41,6.18) \\
\mathrm{n}=55\end{array}$ & $\begin{array}{l}2.51(1.53,7.68) \\
\mathrm{n}=53\end{array}$ & - & - \\
\hline
\end{tabular}

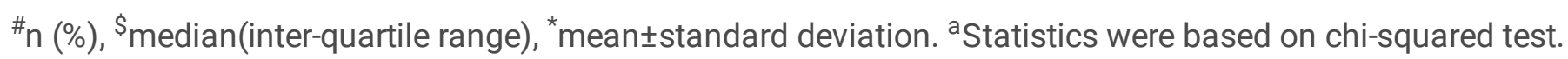
bStatistics were based on Kruskal-Wallis H-test. 'Statistics were based on Mann-Whitney U test. UC history is expressed in years of diagnosed UC. BMI, body mass index; PHQ-9, Patient Health Questionnaire-9; GAD-7, Generalized Anxiety Disorder Scale-7; 5-ASA, 5-aminosalicylic acid.

About half of the UC discovery group met the depression or anxiety criteria (Table 1), of which moderate to severe patients accounted for $42 \%$ (for depression) and $21 \%$ (for anxiety). $13 \%$ of UC patients in the discovery cohort had thoughts of suicide. About one third of MDD patients took antidepressant/anxiolytic drugs in recent three months, while none of UC patients took medication for their mental problems.

\section{Special fecal microbiota features of active UC patients with depression and anxiety}

A total of 3777 operational taxonomic units (OTUs) was detected in fecal samples. After quality control, 296, 276, and 344 OTUs of UC discovery group, replication group, and non-IBD group (HC and MDD) were retained for subsequent analyses.

Among the host characteristics, depression and anxiety levels had moderate impact on the gut microbiota structure of UC patients (Fig. 2A). Compared with UC patients without depression or anxiety (UCND/UCNA), UC patients with depression or anxiety (UCD/UCA) had lower fecal microbial abundance with lower alpha-diversity, illustrated by reduced Shannon index and PD whole tree diversity (Fig. 2B). The composition of fecal microbiota differed among the four different groups (Fig. 2C, Additional file 1: Figs.S1,S2), exhibiting in the differential abundances of major families, including decreased Prevotellaceae and increased Bacteroidaceae in the patients with depression and anxiety.

In the discovery cohort, 50 OTUs, belonging to 32 genus and species, showed differences in relative abundance in UCD/UCA, compared to UCND/UCNA (Additional file 2: Table S2,S3). 20 genus of them further showed inter-group differential abundances at the genus level (Mann-Whitney U test or Student's t-test: $p<0.05$; Fig. 2D, Additional file 2: Table S4, S5). The abundance of OTU_1989, which belonged to Bifidobacterium, was significantly enriched in UCA group after multiple testing corrections (Bonferroni-corrected $p=0.020$ ), though not replicated. The less OTU_1353, which belonged to Prevotella_9, harbored by UC patients with anxiety wasreplicated with nominal significance $(p=0.027)$. Both UCD and UCA groups harbored significantly less Collinsella and Prevotella_9 in the discovery cohort, and this trend remained consistent in the replication cohort (Fig. 2D). Further investigation at the taxonomy level revealed a successfully replicated enrichment of Sellimonas and depression $\left(P_{\text {discovery }}=0.018\right.$, Bonferroni-corrected $\mathrm{P}_{\text {replication }}=0.021$ ).

As PHQ-9(Patient Health Questionnaire-9) and GAD-7(Generalized Anxiety Disorder Scale-7) depict depression and anxiety levels by five-level classification, the associations between gut microbiota and mental state were further investigated by analysis of variance (ANOVA). In the discovery cohort, 12 OTUs showed significant differences between different depression/anxiety levels after multiple testing corrections $\left(p<8.4 \times 10^{-5}\right.$, ANOVA, Additional file 2: 
Table S6, S7). The differential abundance of OTU_105: Streptococcus at different anxiety levels could be successfully replicated (Bonferroni-corrected $p=0.042$ ). Further investigation at the taxonomic level confirmed the significance of 37 kinds of bacteria (Additional file 2: Table S8, S9). Among them, the associations of Peptoclostridium and Bacilli with depression levels were replicated (nominal $p<0.05$, Additional file 1: Figure S3).

Since most mental disorders are indeed mental disturbance on a continuous scale, we additionally conducted linear regression analysis and non-parametric Spearman's rank correlation test between relative abundanceof gut microbiota and PHQ-9/GAD-7 scores (Fig. 2E). The positive correlations between OTU_2853: Clostridium sensu stricto_ 1 and depression /anxiety, and between OTU_105: Streptococcus and anxiety, were significant in both the discovery and the replication cohorts by linear regression (replication $p<0.05$, Additional file 2: Table S10). There is significantly negative Spearman's rank correlation between OTU_2239: Lachnospiraceae abundance and PHQ-9 /GAD-7 scores, and between OTU_1353: Prevotella_9 abundance and GAD-7 scores in both the discovery and the replication cohorts (replication $p<0.005$, Additional file 2: Table S11). On the taxonomic scale, with the abundances of Lactobacillales and Bacilli increasing, UC patients' anxiety levels elevated (discovery: $p<0.001$; replication: FDR = 0.09 and 0.16 for Bacilli and Lactobacillales, Additional file 2: Table S12, S13). Enterococcus, which belongs to Bacilli, was also positively correlated with depression and anxiety levels; on the other hand, Dorea,

Erysipelotrichaceae UCG_003, and Ruminococcus gauvreauii group were negatively correlated with depression and anxiety levels in both UC patients and non-IBD people (Fig. 2D and 2E). In addition, the negative association of Prevotella_9 with depression and anxiety remained consistent in both discovery and replication cohorts (Fig. 2D and 2E).

Finally, a total of six analysis methods including both parametric and non-parametric methods were considered together to screen out gut microbiota consistently associated with the mental phenotypes. 26 OTUs in the discovery cohort were revealed to be associated with depression/anxiety levels by at least three methods. Among them, OTU_2853: Clostridium sensu stricto_1, OTU_2729: Lachnoclostridium, OTU_2626: Anaerotruncus, OTU_1353: Prevotella_9, 0TU_1080: Prevotella_9 could be nominally replicated $(p<0.05)$ by at least one method in the replication cohort. On the taxonomic scale, 17 genus and four species were associated with both depression and anxiety levels in UC patients, suggested by at least one analysis method (Fig. 2F); among which, Dorea was revealed by the most analyses.

To explore the functional implications of the microbiota shift that drive UC patients to be depressive and anxious, microbial functional pathways were investigated with PICRUSt. 257 Kyoto Encyclopedia of Genes and Genomes (KEGG) pathways presented in at least $40 \%$ samples were identified, 30 pathways differed between UCD/UCA and UCND/UCNA (Additional file 2: Table S14). These pathways mainly involved amino acids biosynthesis, metabolism of bioactive molecules such as cofactors and vitamins, DNA repair, protein translation and degradation. Lysine biosynthesis was up-regulated in UCA and MDD (Additional file 2: TableS14, S15). Nicotinate and nicotinamide metabolism, one carbon pool by folate, and proteasome were down-regulated in UCA in both cohorts (Fig. 2G). In addition, Prevotella_9 was significantly negatively correlated with the above pathways involving metabolism of amino acids, cofactors and vitamins ( $p<0.01$, Spearman's rank correlation test).

As a functional community, gut bacteria interact with each other dynamically to form a topological network. The gut microbial network of UCD harbored less co-occurring and more co-excluding connections than that of UCND. Similar reduced network complexity was observed in the replication cohort (Additional file 1: Figure S3, S4). UC patients who developed depression and anxiety established new gut microbial networks. Akkermansia, Aggregatibacter, Enterococcus, Alloprevotella, and Collinsella formed a co-occurring network in UCND. Most bacteria in this network 
were enriched individually in UCD, but their connections diminished (Fig. 2H and 2I). Sellimonas (which was enriched in depressed and anxious UC patients) changed its co-occurrence with Dorea (which was negatively correlated with depression and anxiety) in UCND to with Haemophilus (which was enriched in depressed and anxious UC patients) in UCD.

\section{Metabolomic Signatures of Active UC Patients with Depression and /or Anxiety}

In order to explore whether there are specific metabolites that bridge the communication between gut microbiota and mental disorders, serum non-targeted metabolomics was performed by high-throughput liquid chromatography /mass spectrometry in the replication cohort. After quality control, 5405 and 4352 peaks were identified in positive ion mode (ES+) and negative ion mode (ES-), respectively, of which 299 compounds can be identified. Only metabolites with significant differences indicated by both parametric tests and non-parametric tests were regarded as candidates.

Compared with UCND /UCNA, glycochenodeoxycholate was enriched in the serum of UCD /UCA (VIP > 1, fold change $>1.5, p$-value $<0.05$, Student's t-test \& Mann-Whitney U test, Additional file 2: Table S16, S17). UC patients with anxiety additionally harbored over three times more stearoyllysophosphatidylcholine (1-Stearoyl-2-hydroxy-snglycero-3-phosphocholine) and thioetheramide-PC (Fig. 3A). In addition, stearoyllysophosphatidylcholine was

positively associated with anxiety level by general linear model and Spearman's rank correlation test; dopamine was positively associated with both depression and anxiety in UC patients (Fig. 3B, Additional file 1: Figure S5, Additional file 2: Table S18, S19). Comprehensive analyses with six methods found that rosolic acid, 1-stearoyl-snglycerol, and glycochenodeoxycholate were positively correlated with depression and anxiety levels, revealed by at least four types of analyses (Fig. 3C, Additional file 2: Table S20, S21).

These phenotype-associated metabolites were involved in 54 KEGG pathways, 26 pathways were enriched in UCD (Additional file 2: Table S22) and UCA (Additional file 2: Table S23). These pathways mainly involve functions related to the immune system and the nervous system, especially related to electrophysiological features of neurons, such as long-term depression, long-term potentiation, and gap junction (Fig. 3D). Most of these associated pathways involve one metabolite, 1-Stearoyl-2-arachidonoyl-sn-glycerol, which was a top associated metabolite by ANOVA and Kruskal-Wallis test (Additional file 2: Table S20, S21). The association of primary bile acid biosynthesis and gap junction with depression level was significant after multiple testing corrections. In addition, there was difference in primary bile acid biosynthesis between UC and healthy control (Additional file 2: Table S24). It is noteworthy that primary bile acid biosynthesis involves glycochenodeoxycholate, a metabolite significantly associated with depression and anxiety levels in UC patients. Tryptophan metabolism was suggested to be upregulated in the analysis for anxiety due to the involvement of anthranilic acid (Additional file 2: Table S20, S21, S23), but its association was not significant.

\section{Specific Proteomic Features of Active UC Patients with Depression and Anxiety}

The gut microbiota communicate with the central nervous system through macromolecules (i.e., proteins) as well as small molecules (i.e., metabolites) in the circulatory system. Therefore, we additionally explored serum proteome via TMT quantitative proteomics detection method to find proteins associated with UC-specific mental disorders. Among the 956 proteins identified, 125 and 136 proteins were up-regulated and down-regulated (significance based on $90 \%$ confidence interval), respectively, in UC patients with depression and anxiety (Additional file 2: Table S25, S26). The expression of Crk-like protein (CRKL) changed the most, with down-regulation of more than 10 times. 26 
proteins were down-regulated by over three times and they interact with each other closely (Fig.3E, Additional file 1: Figure S6).

Pathway analysis found these differentially expressed proteins significantly $(q<0.05)$ enriched in 196 gene ontology (GO) - biological process pathways and six KEGG pathways (Fig. 3F, Additional file 2: Table S27). Among them, 59 pathways which mainly involved in immune response (i.e., cytokine production) and neuronal connections (axonal fasciculation) were not significantly regulated in UC patients when compared to healthy control (Additional file 2: Table S28), which might be specific to UC-related mental disturbance. It is of note that pathways involved in inflammatory response and phagocytosis were significantly down-regulated in UC patients with depression and anxiety, while those pathways about axonal pruning were up-regulated a bit. Plasminogen activation and blood coagulation were up-regulated, while scavenging of heme from plasma was mainly down-regulated (Fig. 3F). This combined together with coagulation activation in UC pathology, may aggravate intestinal inflammation.

\section{Integrative Crosstalk of Multi-omics}

There was strong correlations between gut microbiota and serum metabolites or proteins (Fig.4A, 4B, Additional file 1: Figure S7). Prevotella_9 is significantly negatively correlated with metabolites 1-stearoyl-sn-glycerol and 1stearoyl-rac-glycerol ( $p<0.01$, Spearman's rank correlation test). By mediation analysis, it was indicated that the effect of Prevotella_9 on UC patients' depression phenotype might be indirectly mediated by 1-stearoyl-rac-glycerol (average causal mediation effect, $p=0.074$ ).

The correlations among multiple omics data and clinical manifestations related to psychological states or UC were further examined by estimating the proportion of shared information among the different types of data through RV coefficient analysis. A moderate proportion of information (58 87\%) was shared between serum metabolome, UCrelated phenotypes, and psychological state (depression or anxiety) (Fig. 4C).

The significantly correlated gut microbiota, serum metabolites and proteins formed a close, interactive, and comprehensive network in the human body (Fig. 4D). This network centered on Prevotella_9 and 1-Stearoyl-snglycerol, and both were highly associated with a set of immune-related proteins. Additionally, Sellimonas, Dorea, Eubacterium ventriosum group, and Peptoniphilus were highly connected nodes. Most bacteria and all proteins in the network were down-regulated when UC patients get depressed or anxious, while most metabolites in the network were up-regulated.

The microbiota metabolic pathways overlapped with the host's serum metabolic pathways exactly on aminoacyltRNA biosynthesis, ascorbate and aldarate metabolism, and nicotinate and nicotinamide metabolism; they also share metabolisms of some amino acids such as lysine, and metabolism related with pentose (a key component of DNA and RNA), although these pathways were not significant in metabolome pathway analyses (Additional file 2: Table S14, S22, S23). Serum metabolites and proteins were involved in similar pathways related to cytokine signaling and immune response, fatty acid metabolism, synapse function, and Fc (epsilon and gamma) receptor signaling (Additional file 2: Tables22, S23, S27).

\section{Shared Mechanisms of UC and Depression}

In non-IBD population, patients with major depression disorder (the MDD group) harbored more Sellimonas (Student's t-test, $p<0.05$ ), less Erysipelotrichaceae UCG_003 (Student's t-test, $p<0.05$ ) and Prevotella_9 (MannWhitney U test, $p<0.05$ ), in consistency with the results in UC population (Additional file 2: Table S29). 
When considering UC as a phenotype, most gut bacteria, about half of serum metabolites and proteins that were associated with UC-related depression/ anxiety also had differential abundance in UC patients when compared with healthy control (Additional file 2: Table S30, S31, S32). On the other hand, there were some gut bacteria, serum metabolites and soluble proteins specifically associated with UC-related depression and anxiety while not correlated with UC phenotype, such as Prevotella_9, Erysipelotrichaceae UCG_003, Ruminococcus gauvreauii group, Dorea, Eubacterium ventriosum group, dopamine, rosolic acid, CRKL and a set of immunoglobulin heavy variable chain proteins.

\section{Discussion}

Here, we report molecular signatures, in terms of gut microbiota, serum metabolomics, and serum proteomics, in active UC with depression and anxiety in 240 participants totally, using a two-step approach with replication study. Previously, there is one publication studied gut microbiota features for Swiss inactive IBD patients with depression, with only one study group of 171 subjects. Compared to it, the current study took a two-step approach with an additional replication cohort and two control groups (healthy control and major depression patients without IBD), leading to a larger sample size of 240; second, not only gut microbiota but also serum metabolome and proteome were investigated, to propose a multi-omics network that could interact with the brain; last, this study employed various statistical methods, including both parametric and nonparametric tests at different levels. All these factors integrated together lead to more reliable discoveries of the current study.

In the current study, UC patients who accompanied by depression and anxiety are found to harbor less Prevotella_9 and Erysipelotrichaceae UCG_003, but more Sellimonas in the feces. In consistency, Prevotella, which has been found to participate in various emotion-related metabolic pathways such as tryptophan and glutamate synthesis[23], is reported to be associated with lower depression and anxiety level, psychological stress and higher quality of life in IBD patients[22]. The abundance of Prevotella is reported to be associated with hippocampus's function and structure[24]. There is few report about the relationship of subjective mood and Erysipelotrichaceae UCG_003 and Sellimonas, which needs further research about their functional significance. Dorea is reported to reduce in abundance in IBD or patients with Parkinson's disease[25,26]. Collinsella belongs to Coriobacteriia, which is reported to be associated with bipolar disorder and the related neural inflammation[27]. Eubacterium ventriosum group was negatively correlated with depression and anxiety levels in the current study, in consistency with previous reports of lower Eubacterium in UC and bipolar patients[28,29]. The increase of Bacteroidaceae in the patients with depression and anxiety is consistent with a previous study that mice monocolonized with Bacteroides fragilis, which could also produce GABA[20], display deficits in serum serotonin[19]. Ruminococcus could predict serum serotonin and cortisol levels[30], and is significantly associated with a depression indicator[31] and IBD[32] and gut inflammation[33]. Lachnospiraceae and Ruminococcaceae, which accounted for $42.62 \%$ of the identified gut microbiota, could produce butyric acid, a subtype of short-chain fatty acids (SCFAs). SCFAs can affect the brain through direct humoral action, indirect hormone, immune and neural pathways[34]. Serum metabolomics identified a type of SCFA, valeric acid, which showed a decreasing trend in UC depression and anxiety group with borderline significance. UC patients with depression and anxiety harbored more 1-stearoyl-rac-glycerol and 1-stearoyl-snglycerol, which belong to glyceride substances, in accordance with the fact that depression is usually accompanied by neural lipid metabolism disorders[35]. It suggests that these glyceryl stearate may play a role in UC-related mental disturbance, through interacting with gut microbiota. It is of note that this study found dopamine in the serum to be associated with depression and anxiety in UC patients, supporting the reliability of this study[36]. 
Though tryptophan metabolism was not suggested in our analysis, one metabolite in its pathway, anthranilic acid, was significantly associated with anxiety levels in UC patients. Anthranilic acid is a secondary metabolite of kynurenine, and is reported to be a biochemical "warning sign" of early-stage depression[37] and a marker for inflammation assessment in the brain[38].

There is an interactive and tight connected network (Fig. 5) among certain gut microbiota, serum metabolites and proteins that are associated with UC-specific depression and anxiety. This network centers on gut bacteria Prevotella_9, Peptoniphilus, Dorea, Sellimonas, and Eubacterium ventriosum group, metabolites 1-stearoyl-snglycerol, 2'-deoxy-D-ribose, dopamine, and indole-3-propionic acid, and a set of immunoglobulin proteins. They may exert on the brain through synergistic or antagonistic interactions. However, this study could not dissolve the causal relationship between multi-omics change and mental disturbance, which requires future research using animal models or clinical intervention.

Both metabolomic and proteomic pathways suggested an involvement of inflammatory response and cytokine signaling, as well as synapse formation and function, in depression and anxiety development in UC patients. This proposes a potential tight link between inflammation and neuronal function. While dysfunctional synaptic transformation is known as one mechanism in the etiology of mood disorders, it would be worthwhile to investigate the role of gut inflammation in synapse pruning in the central nervous system and its subsequent influence on subject's general mood. Another noticeable overlap between serum metabolome and proteome is the Fc gamma receptor signaling pathway and phagocytosis mediated by it. The well-known phagocytes in the brain is microglia, which respond to cytokines and metabolic signals in the brain, and play actively in synaptic pruning[39]. Microglia are the major cells expressing complement protein 3 (C3) in the brain. Among the significantly changed genes from the top pathways associated with UC-related depression and anxiety, C3 was revealed the most in the current study. It has been demonstrated that microglia could opsonize and engulf competing synaptic elements in mice's developing nervous system via C3 and its receptors[40]. Furthermore, it is reported that microglia could be regulated by gut microbiota and the produced metabolites. The bacterial metabolites of tryptophan, such as indole-3propionic acid, could act directly on microglia in the brain through activating the aryl hydrocarbon receptor in microglia and lead to irregular synaptic pruning[41]. The amount of indole-3-propionic acid in the serum was revealed to be associated with depression and anxiety levels with an over five folds of increase in UC patients with depression and anxiety (Additional file 2: Table S18, S20), in consistent with the up-regulated synaptic pruning. Indole-3-propionic acid has the potential to treat Alzheimer's disease, but its relationship with depression remains still unclear[42], which is worthy further research. Considering the evidences above, gut inflammation caused by UC might act on microglia in the brain through serum metabolites and proteins, and then affect synapse pruning of microglia, leading to abnormal neural circuits and the overall mood disorders.

There are several limitations in this study. First, the relatively small sample size may produce large variations in the results. Second, it is a cross-sectional observational study, so that causal relationship could not be revealed. Future validation using germfree animal models is needed. Third, the assessment of mental symptoms through questionnaires cannot replace the clinical diagnosis of mental diseases, and the diagnosis could vary due to self uncertainty and carelessness of subjects, thus the actual UC-related depression and anxiety incidence and levels in the current study may be inaccurate. Fourth, this study only includes active UC patients, which is not enough to summarize the histological characteristics of IBD with depression and anxiety.

The incidence of depression and anxiety in active UC patients reached over $50 \%$ in this study. However, the antidepressant /anxiolytic drug use in UC patients is rare, and most patients will not actively report their emotion

Page $11 / 27$ 
states other than IBD conditions for medical assistance. Thus, it is needed to make clinicians getting aware of the potential of mental disorders for UC patients and to provide treatments accordingly in time.

\section{Conclusion}

Ulcerative colitis patients are accompanied by a high proportion of mental disorders, such as anxiety and depression, especially during the active stage. Ulcerative colitis and depression are independently reported to be related to gut microbiota imbalance. Patients with active ulcerative colitis accompanied by depression and anxiety have lower fecal microbial abundance with more Sellimonas but less Prevotella_9, Erysipelotrichaceae UCG_003, Collinsella, and Dorea. Most metabolites, such as indole-3-propionic acid, glycochenodeoxycholate and glyceryl stearate, increased, while 2'-deoxy-D-ribose and a set of immunoglobulin family decreased in the serum of UC patients accompanied by depression and anxiety. These gut bacteria, serum metabolites and proteins significantly associated with UC-related depression and anxiety are closely correlated with each other, forming a highly connected multi-omic network, centring on Prevotella_9 and 1-stearoyl-sn-glycerol. This network might affect the neuronal refinement (i.e., synaptic pruning) in specific mood-related brain regions via acting on microglia, either directly by bacteria derived metabolites (i.e., indole-3-propionic acid and SCFAs), or indirectly through influencing certain serum metabolites (i.e., glycochenodeoxycholate and glyceryl stearate) and systematic inflammation state. These findings propose potential targets (bacteria, metabolites, proteins) for auxiliary diagnosis and clinical intervention of mental disorders in patients with active ulcerative colitis.

\section{Materials And Methods}

\section{Study design and population}

This study was approved by the Institutional Review Board of the Affiliate Hospital of Nanjing University of Chinese Medicine, and was performed in accordance with the principle of the Helsinki Declaration II. A written informed consent was obtained from each participant.

This is a single-center, prospective and observational cross-sectional study, including two cohorts: discovery cohorts and replication cohorts. The discovery cohorts included three groups of subjects, all aged between 18 and 65 years old: active UC patients, non-IBD anxiety and depression patients, and healthy subjects. The replication cohort only includes active UC patients, with the same inclusion and exclusion criteria as the discovery cohort. Demographic information, mental health measurements, dietary habits, and Bristol stool scores of all subjects were collected. UC patients also reported their disease types, severity of disease, duration of disease, medication and laboratory examination results.

UC patients in active period were recruited in the outpatient department and ward of digestive department at the Jiangsu Province Hospital of Chinese Medicine; all participants were diagnosed with UC; the active period was defined as Mayo score 2 points, and Mayo endoscopy score $\geq 1$ point was satisfied. Non-IBD depression and anxiety patients were recruited in the psychological clinic of Jiangsu Province Hospital of Chinese Medicine. These patients complained of anxiety, depression and other emotional disorders. Healthy subjects matched in age and sex and not accompanied by emotional disorders such as depression and anxiety were recruited.

The current gastrointestinal symptoms, IBD family history, malignant tumors and autoimmune diseases of non-IBD anxiety and depression patients and healthy subjects were excluded through interviews and questionnaires. Patients unable to understand or provide informed consent, and those who did not have a confirmed diagnosis of 
IBD in their medical records were excluded. Consented patients provided demographic information. All groups of subjects did not use any form of antibiotics within 4 weeks before collecting samples. UC patients did not receive enema treatment within half a month, and other therapeutic drugs were not restricted. Patients with systemic infectious diseases, major gastrointestinal surgery history and other types of mental disorders were excluded. Diet was not controlled.

\section{Definition of Normal and Abnormal Anxiety and Depression}

Patient Health Questionnaire-9 (PHQ-9)[43] was used to evaluate the depression level for each subject. It is a nineitem, self-report questionnaire that assesses symptoms of depression. Each item of the PHQ-9 maps onto DSM-IV major depression criteria, PHQ-9 has high specificity and sensitivity in the screening of depression. The total score of PHQ-9 is between 0 and 27, and the critical score is 5, 10, 15, 20, i.e. 0-4, 5-9, 10-14, 15-19, 20-27, representing non-depression, mild, moderate, moderate, severe depression, respectively. A PHQ-9 score of $\geq 5$ is consistent with at least mild depression. A score of $\geq 10$ is consistent with moderate to severe depression.

The anxiety level of the subjects was evaluated by using the Generalized Anxiety Disorder Scale-7 (GAD-7)[44]. It was developed as a screening tool for detecting GAD in primary care patients and has become a widely used measure in adults. It includes 7 questions, with a total score of $0-21$, and a critical score of 5,10,14,19, i.e. 0-4,5-9,1013,14-18,19-21, representing non-anxiety, mild, moderate, moderate, severe anxiety, respectively.

In our study, PHQ-9 and GAD-7 scores $\geq 5$ were defined as depression/anxiety, respectively. Non-IBD anxiety and depression group should meet PHQ-9 scores $\geq 5$ and GAD-7 scores $\geq 5$, while healthy subjects should meet PHQ-9 scores $<5$ and GAD-7 scores $<5$.

\section{Sample Preparation}

All participants received a stool sample collection kit (Beijing Allwegene Technology Co., Ltd., China), with illustrations of the collection procedure explaining the operation details to the patient in detail so as to minimize possible contamination. Fecal samples were transferred to $-80^{\circ} \mathrm{C}$ for storage within 2 hours after collection until further sequencing. $5 \mathrm{ml}$ of fasting serum from all UC patients in the replication cohort were collected and stored at $-80^{\circ} \mathrm{C}$ for serum non-target metabolomics and serum proteomics analysis.

\section{High-throughput 16S rRNA Gene Sequencing}

DNA was extracted using E.Z.N.A.R Stool DNA Kit (Omega Bio-tek, Norcross, GA, U.S.A.) following the manual. Purity and quality of the genomic DNA were checked on $0.8 \%$ agarose gels.

The V3-4 hypervariable region of bacterial 16S rRNA gene were amplified with the primers 338F (ACTCCTACGGGAGGCAGCAG) and 806R (GGACTACHVGGGTWTCTAAT)[45]. For each fecal sample, 8-digit barcode sequence was added to the $5^{\prime}$ end of the forward and reverse primers (provided by Beijing Allwegene Technology Co., Ltd., China). Polymerase chain reaction (PCR) was carried out on a Mastercycler Gradient (Eppendorf, Germany) using $25 \mu$ l reaction volumes, containing $12.5 \mu$ KAPA 2G Robust Hot Start Ready Mix, $1 \mu$ l forward primer $(5 \mu \mathrm{M}), 1$ $\mu \mathrm{l}$ reverse primer $(5 \mu \mathrm{M}), 5 \mu \mathrm{l}$ DNA (total template quantity is $30 \mathrm{ng}$ ), and $5.5 \mu \mathrm{l} \mathrm{H}_{2} \mathrm{O}$. Cycling parameters were $95^{\circ} \mathrm{C}$ for $5 \mathrm{~min}$, followed by 28 cycles of $95^{\circ} \mathrm{C}$ for $45 \mathrm{sec}, 55^{\circ} \mathrm{C}$ for $50 \mathrm{sec}$, and $72{ }^{\circ} \mathrm{C}$ for $45 \mathrm{sec}$ with a final extension at $72{ }^{\circ} \mathrm{C}$ for 10 min. PCR products were purified using a QIAquick Gel Extraction Kit (QIAGEN, Germany), quantified using real-time quantitative PCR, and sequenced at Beijing Allwegene Technology Co., Ltd. (China). 
Deep sequencing was performed on Miseq platform at Beijing Allwegene Technology Co., Ltd. (China) and then analysed using Illumina Analysis Pipeline Version 2.6.

Raw data were first screened and sequences were removed from further consideration if they were shorter than 200 bp, had a low quality score $(\leq 20)$, contained ambiguous bases, or did not exactly match to primer sequences and barcode tags. Qualified reads were separated using the sample-specific barcode sequences and trimmed with Illumina Analysis Pipeline Version 2.6. The dataset were then analyzed using QIIME. Sequences were clustered into OTUs at a similarity level of $97 \%[46]$, to generate rarefaction curves and to calculate the richness and diversity indices. Only those OTUs with a relative abundance above $0.5 \%$ of total sequences in at least one sample were kept. The Ribosomal Database Project Classifier tool was used to classify all sequences into different taxonomic groups against with SILVA 128 database[47].

To examine similarity between different samples, clustering analyses and principal component analysis (PCA) were performed based on the OTU information from each sample using R[48]. The evolution distances between microbial communities from each sample were calculated using the unweighted unifrac algorithms and represented as an Unweighted Pair Group Method with Arithmetic Mean clustering tree describing the dissimilarity (1-similarity) between multiple samples[49]. A Newick-formatted tree file was generated through this analysis. PICRUSt(Phylogenetic Investigation of Communities by Reconstruction of Unobserved States) analysis was conducted to predict pathways of those taxa significantly enriched in each group as decribed previously[50].

\section{Metabolomics Profiling of Human Serum Samples}

Fasting blood samples were collected in $5 \mathrm{~mL}$ vacutainer tubes containing the chelating agent ethylene diamine tetraacetic acid (EDTA), then the samples were centrifuged for $15 \mathrm{~min}\left(1500 \mathrm{~g}, 4^{\circ} \mathrm{C}\right)$. Each aliquot $(150 \mu \mathrm{L})$ of the plasma sample was stored at $-80{ }^{\circ} \mathrm{C}$ until analysis. The plasma samples were thawed at $4{ }^{\circ} \mathrm{C}$ and $100 \mu \mathrm{L}$ aliquots were mixed with $400 \mu \mathrm{L}$ of cold methanol/acetonitrile $(1: 1, v / v)$ to remove the protein. The mixture was centrifuged for $15 \mathrm{~min}\left(14000 \mathrm{~g}, 4^{\circ} \mathrm{C}\right)$. The supernatant was dried in a vacuum centrifuge. For LC-MS analysis, the samples were re-dissolved in $100 \mu \mathrm{L}$ acetonitrile/water $(1: 1, \mathrm{v} / \mathrm{v})$ solvent. To monitor the stability and repeatability of instrument analysis, quality control (QC) samples were prepared by pooling $10 \mu \mathrm{L}$ of each sample and analyzed together with the other samples. The QC samples were inserted regularly and analyzed in every 5 samples.

Metabolic profiling of samples was performed on an Agilent 1290 Infinity LC system (Agilent Technologies, SantaClara, California, USA) coupled with an AB SCIEX Triple TOF 6600 System (AB SCIEX, Framingham, MA, USA) in Shanghai Applied Protein Technology Co., Ltd (China). Chromatographic separation was implemented on ACQUITY UPLC BEH Amide $1.7 \mu \mathrm{m}(2.1 \times 100 \mathrm{~mm})$ columns for both positive and negative models. The column temperature was set at $25^{\circ} \mathrm{C}$. The mobile phase contained an aqueous solution of $25 \mathrm{mM}$ ammonium acetate and $25 \mathrm{mM}$ ammonium hydroxide (A) and acetonitrile (B). The gradient was $95 \% \mathrm{~B}$ and $5 \% \mathrm{~A}$ for $1 \mathrm{~min}$, with a linear reduction to $65 \% \mathrm{~B}$ and $35 \%$ A over $13 \mathrm{~min}$, a reduction to $40 \% \mathrm{~B}$ and $60 \%$ A over $2 \mathrm{~min}$, maintenance for $2 \mathrm{~min}$ and an increase to $95 \% \mathrm{~B}$ and $5 \%$ A over $0.1 \mathrm{~min}$, with a 5 -min re-equilibration period. The delivery flow rate was $300 \mu \mathrm{L} / \mathrm{min}$, and 2 $\mu \mathrm{L}$ aliquot of each sample was injected onto the column.

For mass spectrometric (MS) detection, the following ESI source conditions were used: ion source gas 1 (Gas1) of $60 \mathrm{psi}$, ion source gas 2 (Gas2) of $60 \mathrm{psi}$, curtain gas (CUR) of $30 \mathrm{psi}$, source temperature of $600{ }^{\circ} \mathrm{C}$, and ion spray voltage floating (ISVF) of $\pm 5500 \mathrm{~V}$. In the MS-only acquisition, the instrument was set to acquire data over the $\mathrm{m} / \mathrm{z}$ range of 60-1000 Da, and the accumulation time for the TOF MS scan was set to $0.20 \mathrm{~s} / \mathrm{spectrum}$. For auto MS/MS acquisition, the instrument was set to acquire data over the m/z range of 25-1000 Da, and the 
accumulation time for the product ion scan was set to $0.05 \mathrm{~s} /$ spectrum. The product ion scan was recorded using information-dependent acquisition (IDA) with the high-sensitivity mode. The parameters were as follows: collision energy (CE): fixed at $35 \mathrm{~V} \pm 15 \mathrm{eV}$; declustering potential (DP): $60 \mathrm{~V}(+)$ and- $60 \mathrm{~V}(-)$; exclude isotopes within $4 \mathrm{Da}$; and the number of candidate ions to monitor per cycle: 10. Quality control (QC) samples were prepared by pooling $10 \mu \mathrm{L}$ of each sample and were analyzed approximately once every 5 injections to monitor the stability and repeatability of the data produced by the instrument.

The raw UPLC-Q-TOF/MS data were converted to $\mathrm{mzXML}$ files using Proteo Wizard MSconventer tool and then processed using XCMS[51] online software. Metabolite structure identification used a method of accurate mass matching and secondary spectral matched against in-house tandem MS spectral library (Shanghai Applied Protein Technology, Ltd, China). The parameters in XCMS were set as follows: centwave settings for feature detection $(\Delta \mathrm{m} / \mathrm{z}=25 \mathrm{ppm}$, peakwidth $=\mathrm{c}(10,60))$; obiwarp settings for retention time correction (profStep =1); and parameters including minfrac $=0.5, \mathrm{bw}=5$ and mzwid $=0.025$ for chromatogram alignment. After being normalized and integrated by using support vector regression, the processed data were uploaded into MetaboAnalyst[52] software for further analysis (www.metaboanalyst.ca). PCA and orthogonal partial least square discriminant analysis (OPLS-DA) were performed for both positive and negative models after log transformation and pareto scaling. The variable importance in the projection (VIP) value of each variable in the OPLS-DA model was calculated to indicate its contribution to the classification. The online KEGG database (http://www.genome.jp/kegg/) (updated: September 14, 2016)[53] was used for the identification of metabolic pathways.

\section{Proteomics Profiling of Human Serum Samples}

Serum pools were depleted of most abundant proteins using Agilent Human-14 Multiple Affinity Removal System Column (Agilent Technologies) following the manufacturer's protocol. The $10 \mathrm{kDa}$ ultrafiltration tube (Sartorius) was used for desalination and concentration of low-abundance components. One volume of SDT buffer was added, boiled for $15 \mathrm{~min}$ and centrifuged at $14000 \mathrm{~g}$ for $20 \mathrm{~min}$. The supernatant was quantified with the BCA Protein Assay Kit (Bio-Rad, USA). The sample was stored at $-80^{\circ} \mathrm{C}$.

For each sample, $20 \mu \mathrm{g}$ of proteins were mixed with $5 \times$ loading buffer and boiled for $5 \mathrm{~min}$. The proteins were then separated on $12.5 \%$ SDS-PAGE gel (constant current $14 \mathrm{~mA}, 90 \mathrm{~min}$ ) and protein bands were visualized by Coomassie Blue R-250 staining for quality control.

After that, $200 \mu \mathrm{g}$ of proteins for each sample were incorporated into $30 \mu \mathrm{l}$ SDT buffer (4\% SDS, 100mM DTT, $150 \mathrm{mM}$ Tris-HCl pH 8.0). The detergent, dithiothreitol (DTT), and other low-molecular-weight components were removed using UA buffer ( $8 \mathrm{M}$ Urea, $150 \mathrm{mM}$ Tris- $\mathrm{HCl} \mathrm{pH} \mathrm{8.0)} \mathrm{by} \mathrm{repeated} \mathrm{ultrafiltration.} 100 \mu$ iodoacetamide (100 mM IAA in UA buffer) was added to block reduced cysteine residues and the samples were incubated for $30 \mathrm{~min}$ in darkness. The filters were washed with $100 \mu \mathrm{l}$ UA buffer three times and then $100 \mu \mathrm{l} 100 \mathrm{mM}$ TEAB buffer twice. Finally, the protein suspensions were digested with $4 \mu \mathrm{g}$ trypsin (Promega, Wisconsin, USA) in 40 $\mu$ I TEAB buffer overnight at $37^{\circ} \mathrm{C}$, and the resulting peptides were collected as a filtrate. The peptide content was estimated by ultraviolet light $(280 \mathrm{~nm})$ using an extinction coefficient of 1.1 of $0.1 \%(\mathrm{~g} / \mathrm{l})$ solution that was calculated based on the frequency of tryptophan and tyrosine in vertebrate proteins.

About $100 \mu \mathrm{g}$ peptide mixture of each sample was labeled using TMT reagent (Thermo Fisher Scientific) according to the manufacturer's instructions. Pierce high pH reversed-phase fractionation kit (Thermo scientific) was used to fractionate TMT-labeled digest samples into 12 fractions by an increasing acetonitrile step-gradient elution according to instructions. 
Each fraction was loaded onto a reverse phase trap column connected to the C18-reversed phase analytical column in buffer A (0.1\% Formic acid) and separated with a linear gradient of buffer B ( $84 \%$ acetonitrile and $0.1 \%$ Formic acid) at a flow rate of $300 \mathrm{~nL} / \mathrm{min}$ controlled by IntelliFlow technology (Thermo Scientific) for nano LC-MS/MS analysis.

Liquid chromatography-mass spectrometry /MS (LC-MS/MS) analysis was performed on a Q-Exactive mass spectrometer (Thermo Scientific) that was coupled to Easy nLC for 60 min in Shanghai Applied Protein Technology Co., Ltd, China. The mass spectrometer was operated in positive ion mode. Mass spectrometric data was acquired using a data-dependent top 10 method dynamically by choosing the most abundant precursor ions from the survey scan $(300-1,800 \mathrm{~m} / \mathrm{z})$ for HCD fragmentation. Automatic gain control (AGC) target was set to $3 \mathrm{E} 6$ and maximum inject time to $10 \mathrm{~min}$. Dynamic exclusion duration was $40 \mathrm{sec}$. Survey scans were acquired at a resolution of 70,000 at $200 \mathrm{~m} / \mathrm{z}$ and resolution for HCD spectra was set to 35,000 at $200 \mathrm{~m} / \mathrm{z}$, and isolation width was $2 \mathrm{~m} / \mathrm{z}$. Normalized collision energy was $30 \mathrm{eV}$ and the underfill ratio, which specifies the minimum percentage of the target value likely to be reached at maximum fill time, was defined as $0.1 \%$. The instrument was run with the peptide recognition mode enabled.

\section{Data Analysis}

Tandem mass spectrometry (MS/MS) spectra were searched using MASCOT engine (Matrix Science, London, UK; version 2.2) embedded into Proteome Discoverer 1.4. Gene ontology (GO) enrichment on one ontologies (biological process) and Kyoto Encyclopedia of Genes and Genomes (KEGG) pathway enrichment analyses were applied based on the Fisher's exact test, considering the whole quantified protein annotations as background dataset. Welch's ttest was applied for the KEGG enrichment analysis of 16S rRNA V3-V4 sequencing analyses. Benjamini-Hochberg correction for multiple testing was further applied to adjust derived $p$-values and significances were considered at $q$ values $<0.05[54]$.

Altogether six analysis method were implemented for gut microbiota and metabolomics analyses, namely, Student's t-test, Mann-Whitney U test, one-way analysis of variance (ANOVA), Kruskal-Wallis test, general linear models (GLM), and Spearman's rank correlation test. For proteomics, Mann-Whitney U test and Student's t-test was implemented with significance suggested by $90 \%$ confidence interval. Major analyses and plotting were implemented with $\mathrm{R}$ version 3.6.2. Multiple testing was corrected in false discovery rate (FDR), set to 0.05.

Genus and species association in each group were estimated using SparCC[55] on selected significantly different genera and species between UC patients with depression/anxiety and non-depression /non-anxiety. Significant co-

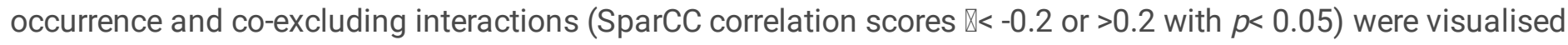
and analysed using igraph package.

The correlation network among multi-omics is realized by Spearman's rank correlation analysis. Mediation analysis was performed to determine the extent to which microbiota mediate the effects of interventions on the UC depression and anxiety phenotype.

\section{Abbreviations}

UC: Ulcerative colitis; IBD: Inflammatory bowel disease; MDD: Major depression disorder, in this study, it refers specifically to the non-IBD depression and anxiety group; BMI: Body Mass Index; PHQ-9: Patient Health Questionnaire-9; GAD-7: Generalized Anxiety Disorder Scale-7; 5-ASA: 5-aminosalicylic acid; OTU: Operational 
Taxonomic Units; UCD: Ulcerative colitis with depression; UCND: Ulcerative colitis without depressionखUCA: Ulcerative colitis with anxiety; UCNA: Ulcerative colitis without anxiety; CRKL: Crk-like protein; SCFAs: Short-chain fatty acids; ANOVA: one-way analysis of variance『KEGG: Kyoto Encyclopedia of Genes and Genomes; GO: Gene Ontology; PCA: Principal component analysis; OPLS-DA: Orthogonal partial least square discriminant analysis; PICRUSt: Phylogenetic Investigation of Communities by Reconstruction of Unobserved States; GLM: General linear models; FDR: False Discovery Rate.

\section{Declarations}

Ethics approval and consent to participate: This study was approved by the Institutional Review Board of the Affiliate Hospital of Nanjing University of Chinese Medicine, Nanjing, China (2018NL-093-02), and informed consent was obtained from all subjects.

Consent for publication: Not applicable.

Availability of data and materials: The access policy and procedures are available through emails to the corresponding authors.

Competing interests: The authors declare that they have no competing interests.

Funding: This study was supported by grants from the Priority Academic Program Development of Jiangsu Higher Education Institutions (PAPD) (ZYXKF020). YC is supported by the Jiangsu "333 High-Level Talent Training Project"second level (SLJ0203).

Authors' contributions: $\mathrm{XY}$ and $\mathrm{BC}$ are joint first authors. $\mathrm{YC}$ obtained funding. YC, $\mathrm{XY}, \mathrm{BC}, \mathrm{GS}, \mathrm{LZ}, \mathrm{BY}$, and JZ and designed the study. XW carefully selected psychological outcome parameters for depression and anxiety questionnaires. HL, BW, and JX collected the data. ZD, ZX, YD and TC collected the fecal samples. SL, HS, and WL collected the serum samples. ZS were involved in data cleaning. $X Y$ and $B C$ analyzed the data and drafted the manuscript. BC, GS, and YC contributed to the interpretation of the results and critical revision of the manuscript for important intellectual content. All authors have read and approved the final manuscript. YC, LZ and GS are the study guarantors.

Acknowledgements: Not applicable.

\section{References}

1. Ng SC, Shi HY, Hamidi N, et al. Worldwide incidence and prevalence of inflammatory bowel disease in the $21 \mathrm{st}$ century: a systematic review of population-based studies. Lancet. 2018;390(10114):2769-2778.

2. Abraham C, Cho JH. Inflammatory bowel disease. N Engl J Med. 2009;361:2066-78.

3. Bernstein CN, Hitchon CA, Walld R, Bolton JM, Sareen J, Walker JR, et al. Increased Burden of Psychiatric Disorders in Inflammatory Bowel Disease. Inflamm Bowel Dis. 2019;25:360-8.

4. Häuser W, Janke KH, Klump B, Hinz A. Anxiety and depression in patients with inflammatory bowel disease: Comparisons with chronic liver disease patients and the general population. Inflamm Bowel Dis. 2011;17:62132.

5. Mikocka-Walus A, Knowles SR, Keefer L, Graff L. Controversies Revisited: A Systematic Review of the Comorbidity of Depression and Anxiety with Inflammatory Bowel Diseases. Inflamm Bowel Dis. 2015;22:752- 
62.

6. Neuendorf R, Harding A, Stello N, Hanes D, Wahbeh H. Depression and anxiety in patients with Inflammatory Bowel Disease: A systematic review. J Psychosom Res. England; 2016;87:70-80.

7. Gracie DJ, Guthrie EA, Hamlin PJ, Ford AC. Bi-directionality of Brain-Gut Interactions in Patients With Inflammatory Bowel Disease. Gastroenterology. United States; 2018;154:1635-1646.e3.

8. Navabi S, Gorrepati VS, Yadav S, Chintanaboina J, Maher S, Demuth P, et al. Influences and Impact of Anxiety and Depression in the Setting of Inflammatory Bowel Disease. Inflamm Bowel Dis. England; 2018;24:2303-8.

9. Yongwen Ng J, Chauhan U, Armstrong D, Marshall J, Tse F, Moayyedi P, et al. A Comparison of the Prevalence of Anxiety and Depression Between Uncomplicated and Complex IBD Patient Groups. Gastroenterol Nurs Off J Soc Gastroenterol Nurses Assoc. United States; 2018;41:427-35.

10. Horst S, Chao A, Rosen M, Nohl A, Duley C, Wagnon JH, et al. Treatment with immunosuppressive therapy may improve depressive symptoms in patients with inflammatory bowel disease. Dig Dis Sci. United States; 2015;60:465-70.

11. Kochar B, Barnes EL, Long MD, Cushing KC, Galanko J, Martin CF, et al. Depression Is Associated With More Aggressive Inflammatory Bowel Disease. Am J Gastroenterol. 2018;113:80-5.

12. Mikocka-Walus A, Pittet V, Rossel J-B, von Känel R. Symptoms of Depression and Anxiety Are Independently Associated With Clinical Recurrence of Inflammatory Bowel Disease. Clin Gastroenterol Hepatol Off Clin Pract J Am Gastroenterol Assoc. United States; 2016;14:829-835.e1.

13. Mikocka-Walus A, Ford AC, Drossman DA. Antidepressants in inflammatory bowel disease. Nat Rev Gastroenterol Hepatol. England; 2020;17:184-92.

14. Farraye FA, Melmed GY, Lichtenstein GR, Kane S V. ACG Clinical Guideline: Preventive Care in Inflammatory Bowel Disease. Am J Gastroenterol. United States; 2017;112:241-58.

15. Lewis K, Marrie RA, Bernstein CN, Graff LA, Patten SB, Sareen J, et al. The Prevalence and Risk Factors of Undiagnosed Depression and Anxiety Disorders Among Patients With Inflammatory Bowel Disease. Inflamm Bowel Dis. England; 2019;25:1674-80.

16. Sharon G, Sampson TR, Geschwind DH, Mazmanian SK. The Central Nervous System and the Gut Microbiome. Cell. 2016;167:915-32.

17. Cryan JF, O'riordan KJ, Cowan CSM, Sandhu K V., Bastiaanssen TFS, Boehme M, et al. The microbiota-gut-brain axis. Physiol Rev. 2019;99:1877-2013.

18. González-Arancibia C, Urrutia-Piñones J, Illanes-González J, Martinez-Pinto J, Sotomayor-Zárate R, Julio-Pieper $M$, et al. Do your gut microbes affect your brain dopamine? Psychopharmacology (Berl). Germany; 2019;236:1611-22.

19. Yano JM, Yu K, Donaldson GP, Shastri GG, Ann P, Ma L, et al. Indigenous bacteria from the gut microbiota regulate host serotonin biosynthesis. Cell. 2015;161:264-76.

20. Strandwitz P, Kim KH, Terekhova D, Liu JK, Sharma A, Levering J, et al. GABA-modulating bacteria of the human gut microbiota. Nat Microbiol. 2019;4:396-403.

21. Takahashi K, Nakagawasai O, Nemoto W, Odaira T, Sakuma W, Onogi H, et al. Effect of Enterococcus faecalis 2001 on colitis and depressive-like behavior in dextran sulfate sodium-treated mice: involvement of the braingut axis. J Neuroinflammation. 2019;16:201.

22. Humbel F, Rieder JH, Franc Y, et al. Association of Alterations in Intestinal Microbiota With Impaired Psychological Function in Patients With Inflammatory Bowel Diseases in Remission. Clin Gastroenterol

Page $18 / 27$ 
Hepatol. 2020;18(9):2019-2029.e11.

23. Valles-Colomer M, Falony G, Darzi Y, Tigchelaar EF, Wang J, Tito RY, et al. The neuroactive potential of the human gut microbiota in quality of life and depression. Nat Microbiol. England; 2019;4:623-32.

24. Labus JS, Hollister EB, Jacobs J, Kirbach K, Oezguen N, Gupta A, et al. Differences in gut microbial composition correlate with regional brain volumes in irritable bowel syndrome. Microbiome. 2017;5:49.

25. Petrov VA, Saltykova I V, Zhukova IA, Alifirova VM, Zhukova NG, Dorofeeva YB, et al. Analysis of Gut Microbiota in Patients with Parkinson's Disease. Bull Exp Biol Med. United States; 2017;162:734-7.

26. Sokol H, Jegou S, McQuitty C, Straub M, Leducq V, Landman C, et al. Specificities of the intestinal microbiota in patients with inflammatory bowel disease and Clostridium difficile infection. Gut Microbes. 2018;9:55-60.

27. Painold A, Mörkl S, Kashofer K, Halwachs B, Dalkner N, Bengesser S, et al. A step ahead: Exploring the gut microbiota in inpatients with bipolar disorder during a depressive episode. Bipolar Disord. 2019;21:40-9.

28. Hirano A, Umeno J, Okamoto Y, Shibata H, Ogura Y, Moriyama T, et al. Comparison of the microbial community structure between inflamed and non-inflamed sites in patients with ulcerative colitis. J Gastroenterol Hepatol. Australia; 2018;

29. Lu Q, Lai J, Lu H, Ng C, Huang T, Zhang H, et al. Gut Microbiota in Bipolar Depression and Its Relationship to Brain Function: An Advanced Exploration. Front psychiatry. 2019;10:784.

30. Mudd AT, Berding K, Wang M, Donovan SM, Dilger RN. Serum cortisol mediates the relationship between fecal Ruminococcus and brain N-acetylaspartate in the young pig. Gut Microbes. 2017;8:589-600.

31. Chahwan B, Kwan S, Isik A, van Hemert S, Burke C, Roberts L. Gut feelings: A randomised, triple-blind, placebocontrolled trial of probiotics for depressive symptoms. J Affect Disord. Netherlands; 2019;253:317-26.

32. Hall AB, Yassour M, Sauk J, Garner A, Jiang X, Arthur T, et al. A novel Ruminococcus gnavus clade enriched in inflammatory bowel disease patients. Genome Med. 2017;9:103.

33. Schirmer M, Garner A, Vlamakis H, Xavier RJ. Microbial genes and pathways in inflammatory bowel disease. Nat Rev Microbiol. 2019;17:497-511.

34. Dalile B, Van Oudenhove L, Vervliet B, Verbeke K. The role of short-chain fatty acids in microbiota-gut-brain communication. Nat Rev Gastroenterol Hepatol. England; 2019;16:461-78.

35. Sowa-Kućma M, Styczeń K, Siwek M, Misztak P, Nowak RJ, Dudek D, et al. Lipid Peroxidation and Immune Biomarkers Are Associated with Major Depression and Its Phenotypes, Including Treatment-Resistant Depression and Melancholia. Neurotox Res. 2018;33:448-60.

36. Grace AA. Dysregulation of the dopamine system in the pathophysiology of schizophrenia and depression. Nat Rev Neurosci. 2016;17:524-32.

37. Sakurai M, Yamamoto Y, Kanayama N, Hasegawa M, Mouri A, Takemura M, et al. Serum Metabolic Profiles of the Tryptophan-Kynurenine Pathway in the high risk subjects of major depressive disorder. Sci Rep. 2020;10:1961.

38. Darlington LG, Forrest CM, Mackay GM, Smith RA, Smith AJ, Stoy N, et al. On the Biological Importance of the 3hydroxyanthranilic Acid: Anthranilic Acid Ratio. Int J Tryptophan Res. 2010;3:51-9.

39. Cowan M, Petri WAJ. Microglia: Immune Regulators of Neurodevelopment. Front Immunol. 2018;9:2576.

40. Stevens B, Allen NJ, Vazquez LE, Howell GR, Christopherson KS, Nouri N, et al. The classical complement cascade mediates CNS synapse elimination. Cell. United States; 2007;131:1164-78.

41. Rothhammer V, Borucki DM, Tjon EC, Takenaka MC, Chao C-C, Ardura-Fabregat A, et al. Microglial control of astrocytes in response to microbial metabolites. Nature. 2018;557:724-8. 
42. Abildgaard A, Elfving B, Hokland M, Wegener G, Lund S. The microbial metabolite indole-3-propionic acid improves glucose metabolism in rats, but does not affect behaviour. Arch Physiol Biochem. England; 2018;124:306-12.

43. Kroenke K, Spitzer RL, Williams JB. The PHQ-9: validity of a brief depression severity measure. J Gen Intern Med. 2001;16:606-13.

44. Löwe B, Decker O, Müller S, Brähler E, Schellberg D, Herzog W, et al. Validation and standardization of the Generalized Anxiety Disorder Screener (GAD-7) in the general population. Med Care. United States; 2008;46:266-74.

45. Munyaka PM, Eissa N, Bernstein CN, Khafipour E, Ghia J-E. Antepartum Antibiotic Treatment Increases Offspring Susceptibility to Experimental Colitis: A Role of the Gut Microbiota. PLoS One. 2015;10:e0142536.

46. Edgar RC. UPARSE: highly accurate OTU sequences from microbial amplicon reads. Nat Methods. United States; 2013;10:996-8.

47. Cole JR, Wang Q, Cardenas E, Fish J, Chai B, Farris RJ, et al. The Ribosomal Database Project: improved alignments and new tools for rRNA analysis. Nucleic Acids Res. 2009;37:D141-5.

48. Wang Y, Sheng H-F, He Y, Wu J-Y, Jiang Y-X, Tam NF-Y, et al. Comparison of the levels of bacterial diversity in freshwater, intertidal wetland, and marine sediments by using millions of illumina tags. Appl Environ Microbiol. 2012;78:8264-71.

49. Jiang X-T, Peng X, Deng G-H, Sheng H-F, Wang Y, Zhou H-W, et al. Illumina sequencing of $16 S$ rRNA tag revealed spatial variations of bacterial communities in a mangrove wetland. Microb Ecol. United States; 2013;66:96104.

50. Langille MGI, Zaneveld J, Caporaso JG, McDonald D, Knights D, Reyes JA, et al. Predictive functional profiling of microbial communities using 16S rRNA marker gene sequences. Nat Biotechnol. 2013;31:814-21.

51. Smith CA, Want EJ, O’Maille G, Abagyan R, Siuzdak G. XCMS: processing mass spectrometry data for metabolite profiling using nonlinear peak alignment, matching, and identification. Anal Chem. United States; 2006;78:779-87.

52. Chong J, Wishart DS, Xia J. Using MetaboAnalyst 4.0 for Comprehensive and Integrative Metabolomics Data Analysis. Curr Protoc Bioinforma. United States; 2019;68:e86.

53. Kanehisa M, Goto S, Sato Y, Furumichi M, Tanabe M. KEGG for integration and interpretation of large-scale molecular data sets. Nucleic Acids Res. 2012;40:D109-14.

54. Hochberg Y, Benjamini Y. More powerful procedures for multiple significance testing. Stat Med. England; 1990;9:811-8.

55. Friedman J, Alm EJ. Inferring correlation networks from genomic survey data. PLoS Comput Biol. 2012;8:e1002687.

\section{Figures}




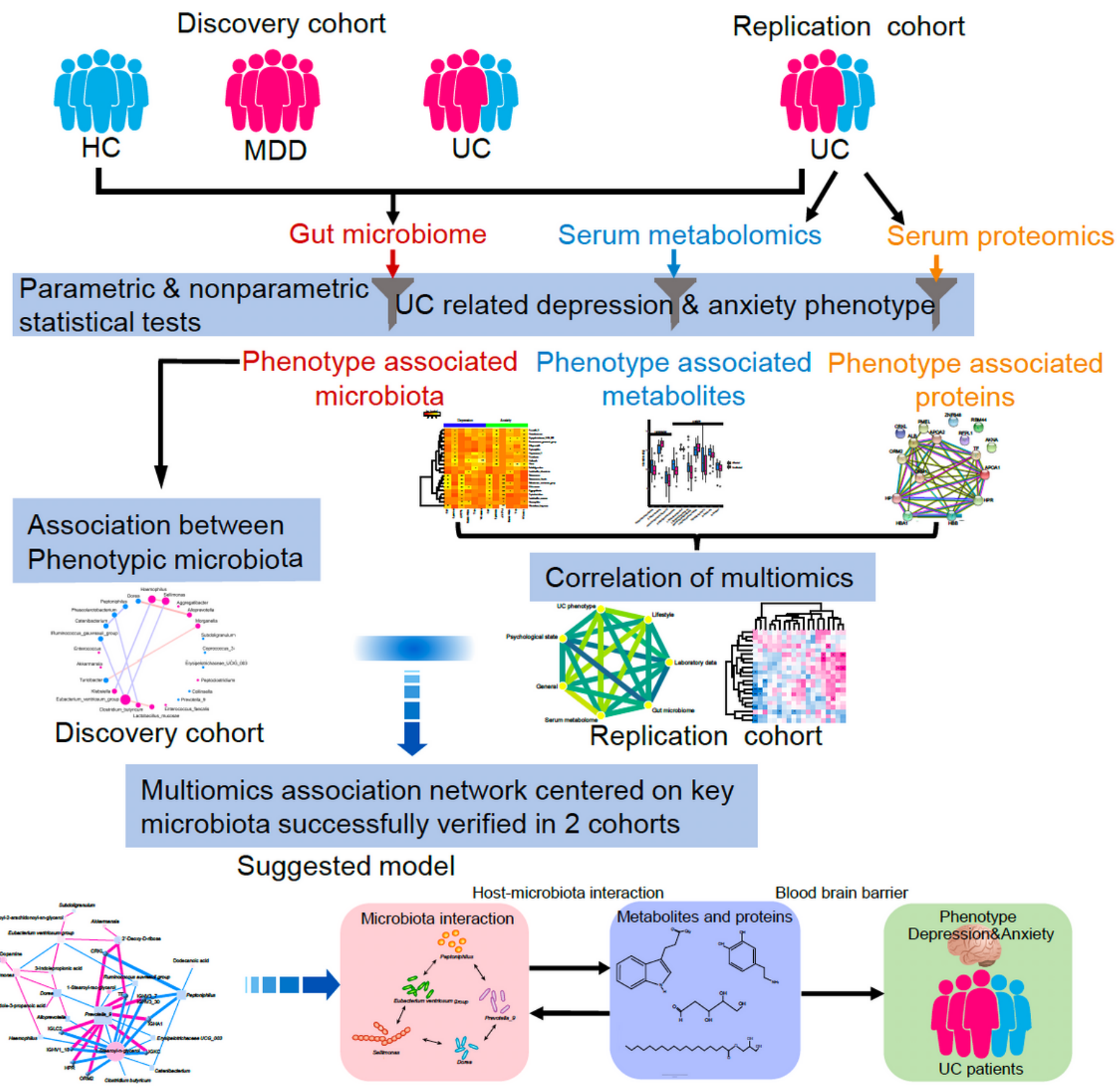

Figure 1

Overview of the workflow integrating human phenotypes, gut microbiota, serum metabolomics and proteomics data. 


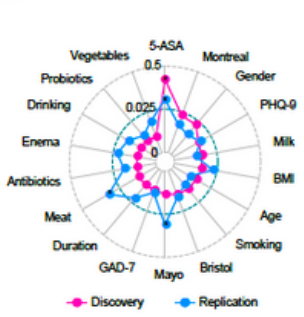

。

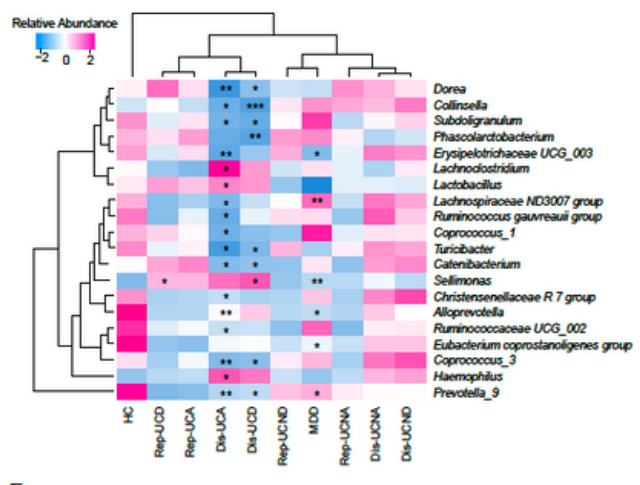

F
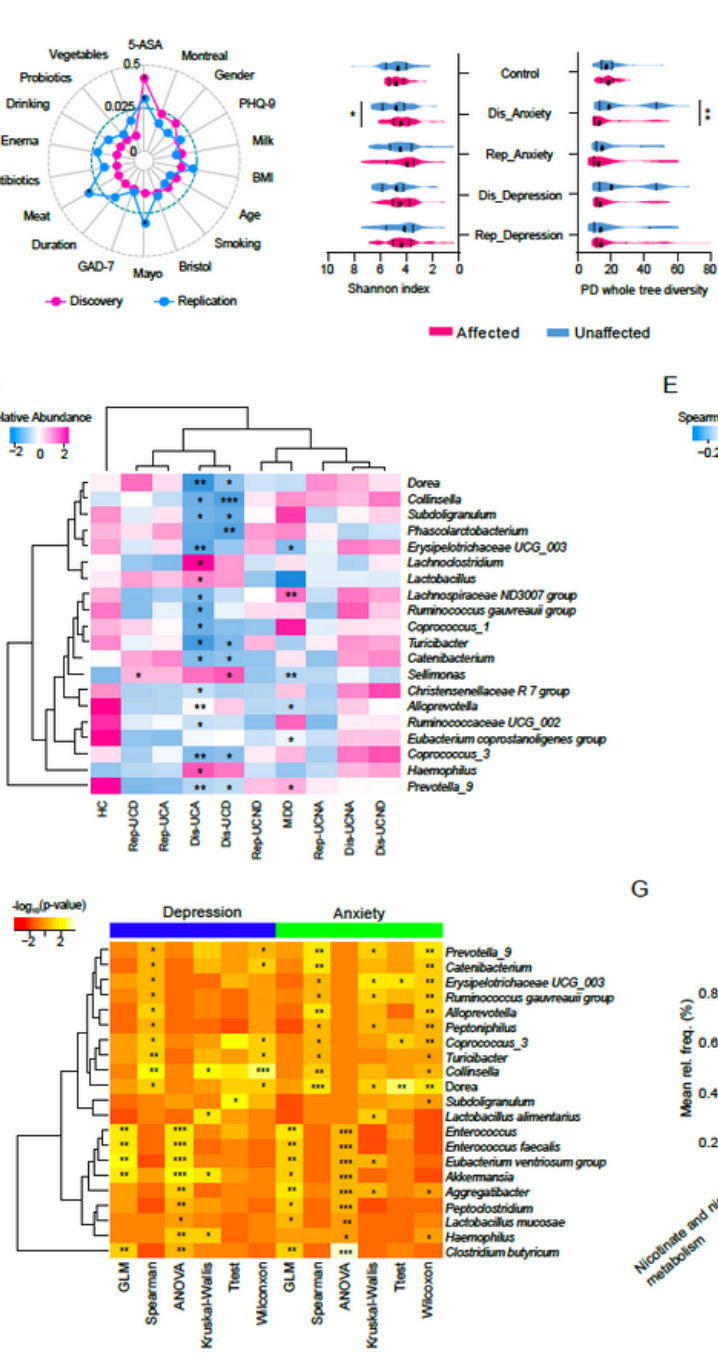

C

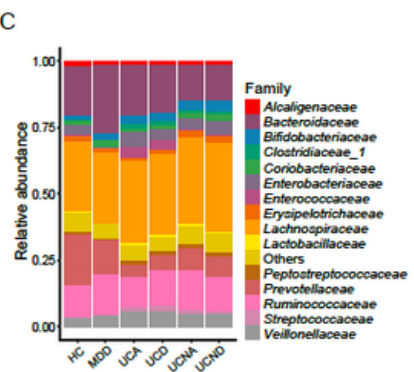

E
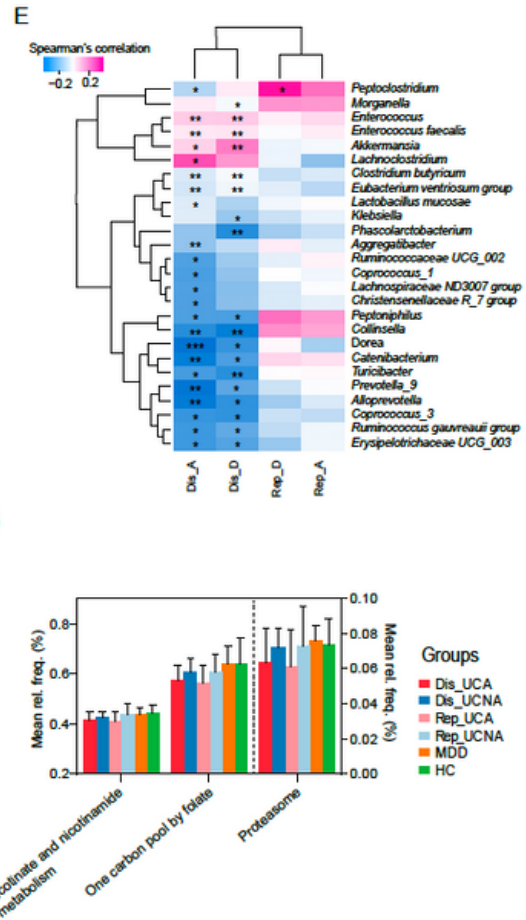

"

I
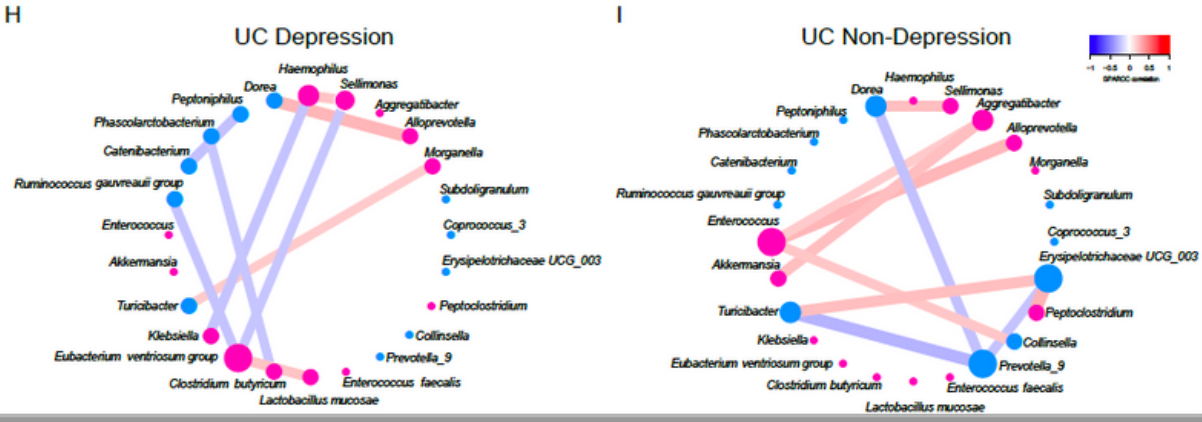

Figure 2

Changes of gut microbiota in UC patients with depression and anxiety. (A) Radar chart illustrates the top host factors that were significantly associated with gut microbial variations. The variations were derived from betweensample unweighted UniFrac distances. Size effects and statistical significance were calculated by PERMANOVA (Adonis). The p-value threshold was set at 0.1. (B) a-diversity (Shannon index and PD whole tree diversity) of gut microbiota in the UC patients are shown, with significance determined by two-tailed Mann-Whitney U test. (C) Composition of gut microbiota in different groups in the discovery cohort is shown in microbial family. The top 15 family were shown. UC group were divided into depression (UCD) and non-depression (UCND) group, or anxiety (UCA) and non-anxiety (UCNA) group, depending on diagnostic criteria. (D) Relative abundances of the genera with statistical differences between UCD/UCA and UCND/UCNA groups, by Student's t-test and Mann-Whitney U test $(p<$ 
0.05). The abundance were scaled based on the taxonomy relative abundance averaged over participants within each group. For each phenotype, significant bacteria determined by either Student's t-test or Mann-Whitney U test, are marked with asterisks. (E) Spearman's rank correlation coefficients between relative abundances of the microbial taxonomy (those with statistical differences between UCD/UCA and UCND/UCNA groups by both general linear regression and Spearman's rank correlation test). Significant correlations (by either general linear regression or Spearman's rank correlation test) are marked as asterisks. (F) Significance (p-values) was shown for each taxonomy, which was significant under either parametric or nonparametric tests $(p<0.05$, any one of 6 kinds of analysis) in both UC depression and anxiety phenotypes. (G) Results of Welch's t-test of PICRUSt-HUMAnN2, showing the three KEGG pathways down-regulated in UCA group in both discovery and replication cohort. Cooccurrence (red) and co-excluding (blue) relationships of genera in UCD (H) and UCND (I) group in the discovery cohort. The edge width and color corresponds to SparCC correlation coefficients. The colour grading indicates the strength of correlation. Bacteria enriched in the UCD group is illustrated in red node, while that enriched in the UCND group is in blue node. Significance levels were indicated by asterisks as * $p<0.05$, ${ }^{*} p<0.01$ and ${ }^{* *} p<0.001$.

A

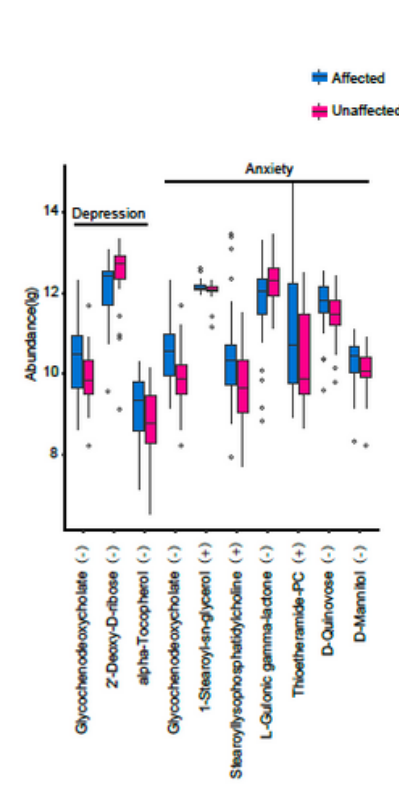

D

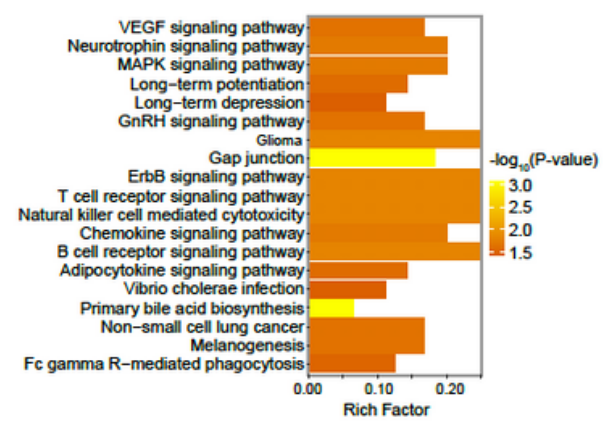

B

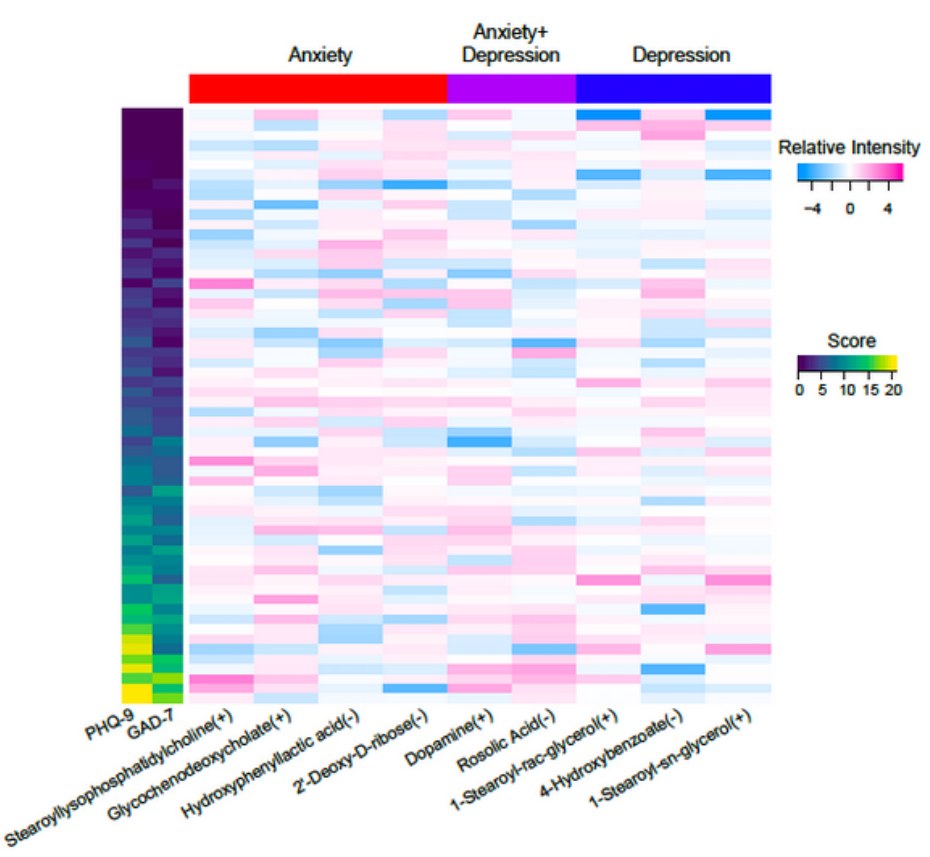

C
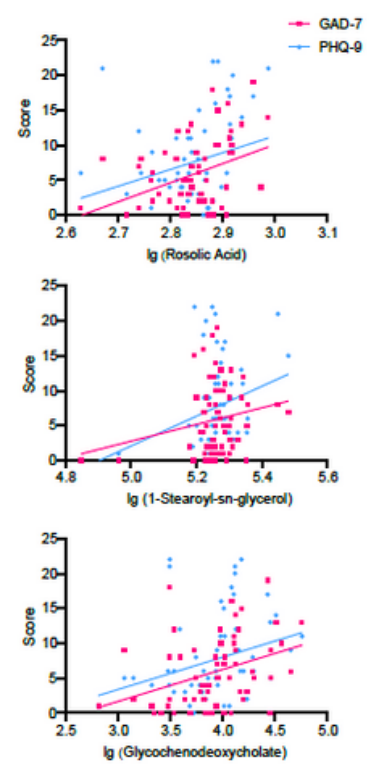

E

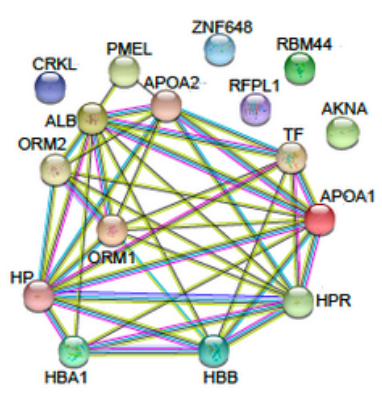

F

\section{Figure 3}

Serum metabolomics and proteomics alterations in UC patients with/without depression and anxiety in the replication cohort $(n=60)$. (A) Differential enrichment of indicated metabolites in UC patients with/without depression and anxiety was illustrated by box plot. Boxes represent the inter-quartile ranges, and lines inside the 
boxes denote medians. Only metabolites with significance in both Student's t-test and Mann-Whitney U test, and VIP $>1$ are shown. (B) Heatmap showing the relative abundance of the metabolites related to UC depression and anxiety phenotype in each subject. The abundance were scaled across each metabolite. Only metabolites with significance in both general linear regression and Spearman's rank correlation test are shown. PHQ-9 and GAD-7 scores were also shown for each subject on the left of the heatmap. (C) Correlations between top associated metabolites and UC depression and anxiety levels (in PHQ-9 and GAD-7 scores) are shown in scatterplots. (D) Metabolomic pathways with statistical enrichment in both UC depression and anxiety phenotype $(p<0.05)$ are shown in bars. The length of bar on the $x$ axis represents the rich factor of KEGG pathways in depression enrichment analysis. The color of bar represents $p$-values for each pathway. (E) Protein-protein interaction network shows the significantly regulated proteins associated with depression and anxiety in UC patients (two-tailed MannWhitney U test, $90 \%$ confidence interval, fold change $<0.3$ or $>3$ ). (F) Selective proteomic pathways with statistical differences $(q<0.001)$ are shown in bar plots. The bar color represents fold changes for the significantly regulated genes in the pathway, with up-regulation in red and down-regulation in blue.

A

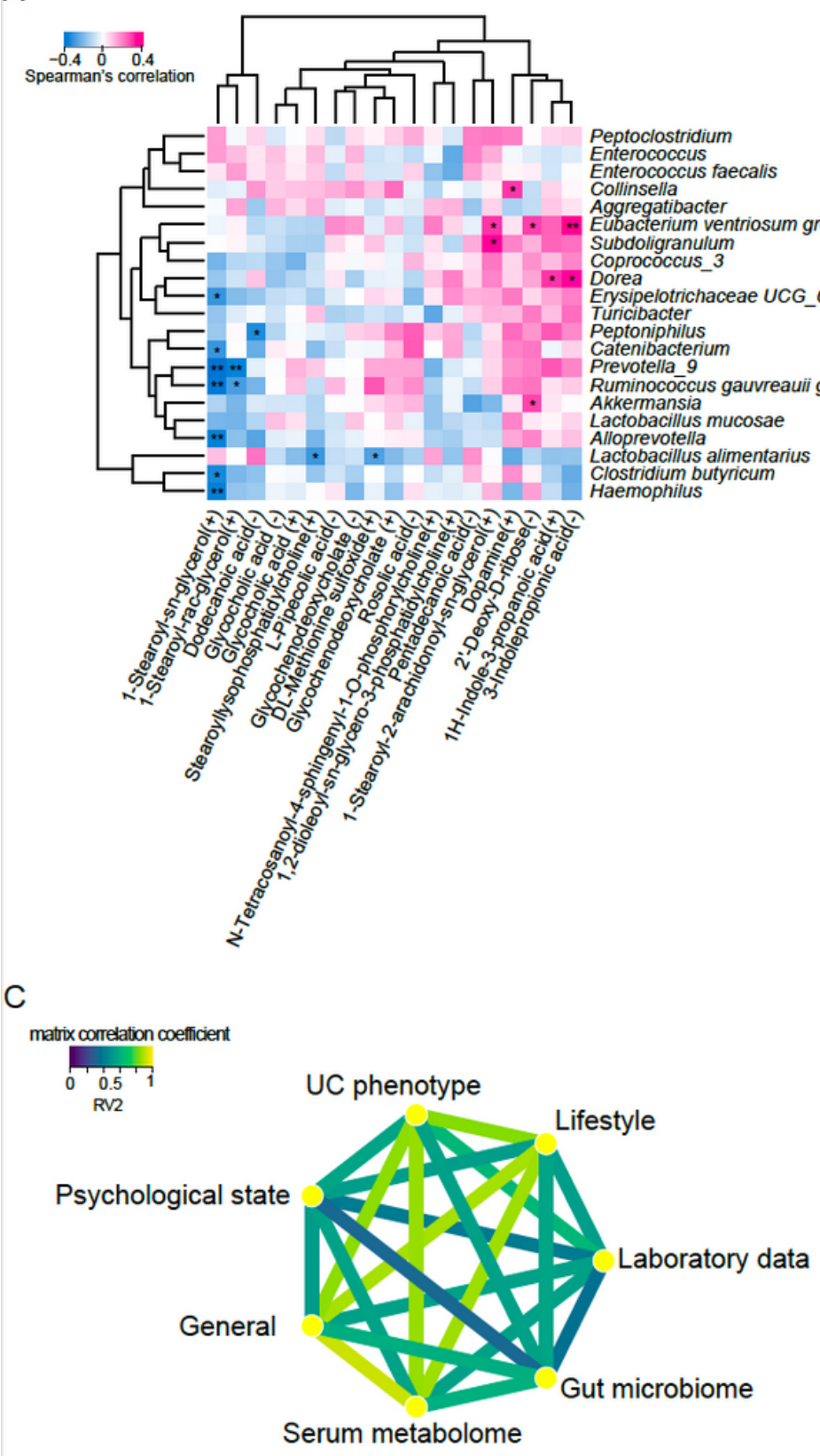

B

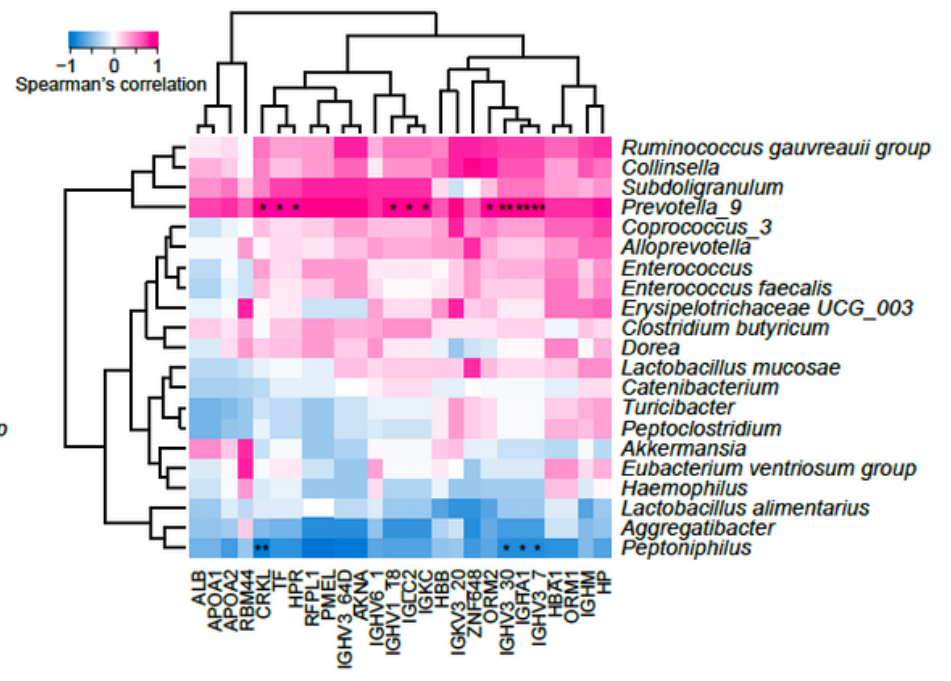

D

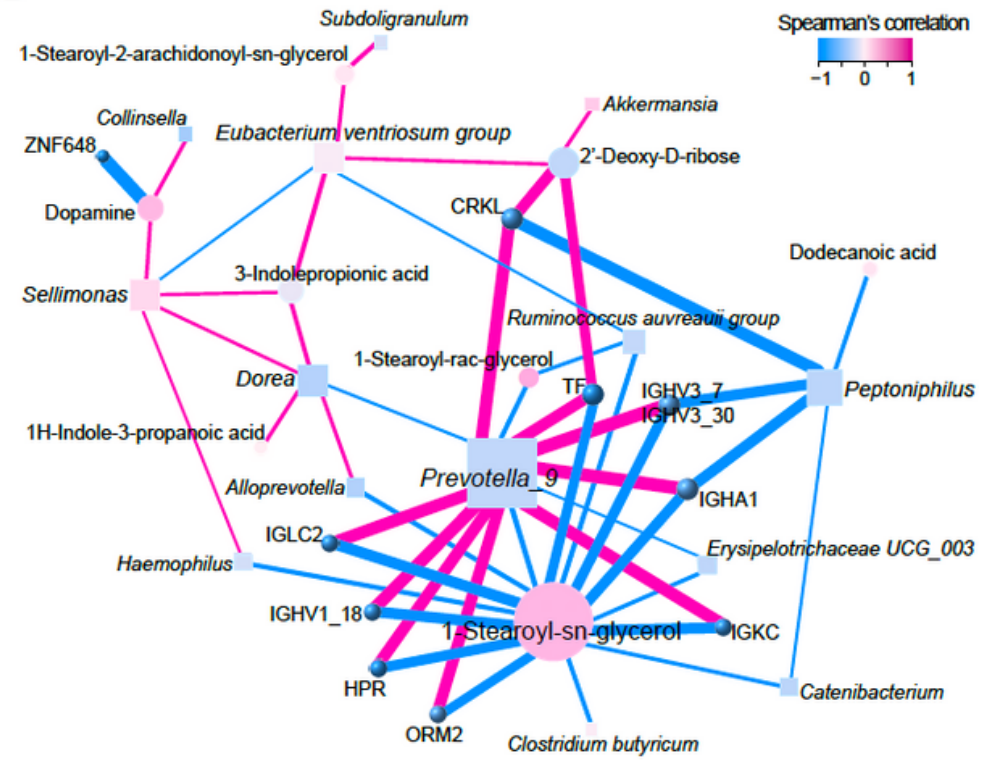




\section{Figure 4}

Integrative data crosstalk and functional characterizations of multi-omics. (A) Spearman's rank correlation coefficients between phenotype-associated microbiota and metabolites in replication cohort. Only microbiota and metabolites with statistical difference (any of the 6 kinds of analysis) for both depression and anxiety phenotype are showed. (B) Spearman's rank correlation between phenotype-associated microbiota and proteins in replication cohort. Only microbiota with statistical difference (any of the 6 kinds of analysis) for both depression and anxiety phenotype and proteins that vary more than 3 times between UCD/UCA group and UCND/UCNA group are showed. (C) Multi-omics phenotype matrix correlation network computed for the patients with matching microbiome and phenomic profiles $(n=60)$ using the modified RV correlation matrix coefficient. Each phenomic table corresponds to a node, and the edges represent the relationships between tables, that is, the percentage of shared information, derived from the RV2 matrix correlation coefficient corresponding to the proportion of variance shared by the two tables, which, like a squared Pearson's correlation coefficient (r2), corresponds to the proportion of the explained variance between two variables. (D) The network diagram shows the interaction among microbiota, metabolites, and proteins. The color of the node represents the correlation between bacteria (or metabolites, proteins) and phenotype. The color of the linked lines represents the correlation between two nodes. Significance levels were indicated by asterisks as ${ }^{*} p<0.05,{ }^{* *} p<0.01$ and $^{* * *} p<0.001$. 


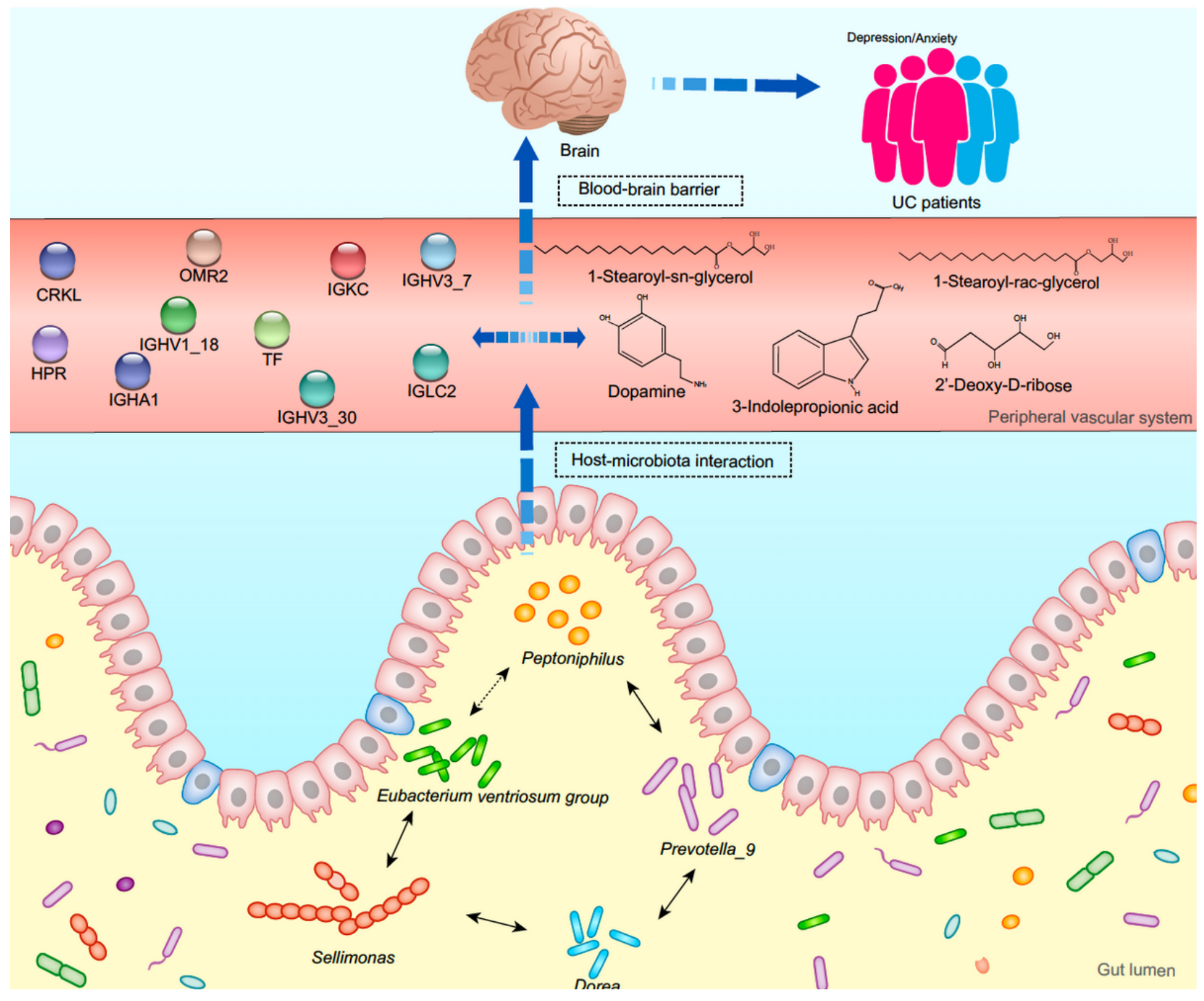

Figure 5

Suggested model of the gut microbiota contribution to serum metabolite and protein levels and UC depression and anxiety phenotype. Serum metabolite and protein levels are influenced by microbiota and their interactions. These changes together may influence neuronal circuitry in the brain and then depression and anxiety phenotype in UC patients.

\section{Supplementary Files}

This is a list of supplementary files associated with this preprint. Click to download.

- Supplementarymicrobiomefinalversion.docx

- Supplementarymicrobiomefinalversion.docx

- Supplementarymicrobiomefinalversion.docx

- Supplementarytablefinalversion.xlsx 
- Supplementarytablefinalversion.xlsx

- Supplementarytablefinalversion.xlsx 